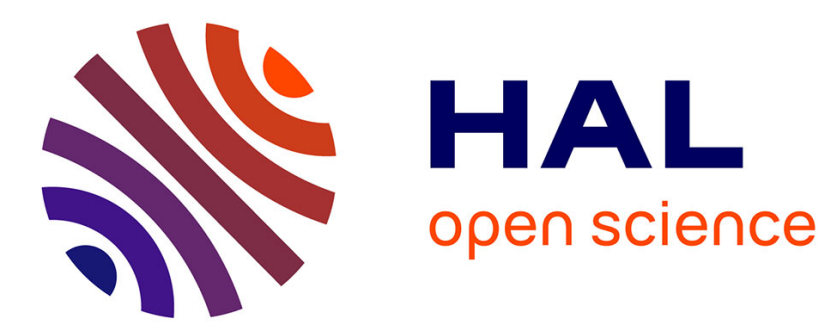

\title{
ON THE THEORETICAL AND NUMERICAL STABILITY OF THE THERMOVISCOELASTIC BRESSE SYSTEM
}

\author{
Maria Inês, M Copetti, E L Arwadi, Wael Youssef
}

\section{- To cite this version:}

Maria Inês, M Copetti, E L Arwadi, Wael Youssef. ON THE THEORETICAL AND NUMERICAL STABILITY OF THE THERMOVISCOELASTIC BRESSE SYSTEM. 2019. hal-02021821

\section{HAL Id: hal-02021821 \\ https://hal.science/hal-02021821}

Preprint submitted on 16 Feb 2019

HAL is a multi-disciplinary open access archive for the deposit and dissemination of scientific research documents, whether they are published or not. The documents may come from teaching and research institutions in France or abroad, or from public or private research centers.
L'archive ouverte pluridisciplinaire HAL, est destinée au dépôt et à la diffusion de documents scientifiques de niveau recherche, publiés ou non, émanant des établissements d'enseignement et de recherche français ou étrangers, des laboratoires publics ou privés. 


\title{
ON THE THEORETICAL AND NUMERICAL STABILITY OF THE THERMOVISCOELASTIC BRESSE SYSTEM
}

\author{
MARIA INÊS M. COPETTI, TOUFIC EL ARWADI, AND WAEL YOUSSEF
}

\begin{abstract}
In this paper, we shall study the stability of the Bresse system where the equations are damped by the dissipation from the viscoelasticity and the thermoelasticity. The thermoviscoelastic Bresse beam is a generalization of the thermoviscoelastic Timoshenko beam. Theoretically, we prove the exponential decay of the energy. Later, we introduce and study an implicit Euler type scheme based on the finite differences in time and the finite elements in space. We show that the discrete energy decreases in time and obtain error estimates. At the end, numerical simulations are presented.
\end{abstract}

\section{INTRODUCTION}

In this work, we consider a new model of thermoviscoelastic Bresse beam which is described by the following equations

$$
\left\{\begin{array}{c}
\rho_{1} \varphi_{t t}-k\left(\varphi_{x}+\psi+l w\right)_{x}-\gamma_{1}\left(\varphi_{x}+\psi+l w\right)_{x t}-k_{0} l\left(\omega_{x}-l \varphi\right) \\
-\gamma_{0} l\left(w_{x}-l \varphi\right)_{t}+l \alpha \theta_{1}=0 \quad \text { in }(0, L) \times(0, \infty), \\
\rho_{2} \psi_{t t}-b \psi_{x x}-\gamma_{2} \psi_{x x t}+k\left(\varphi_{x}+\psi+l \omega\right) \\
+\gamma_{1}\left(\varphi_{x}+l \omega+\psi\right)_{t}+\alpha \theta_{2 x}=0 \quad \text { in }(0, L) \times(0, \infty), \\
\rho_{1} \omega_{t t}-k_{0}\left(\omega_{x}-l \varphi\right)_{x}-\gamma_{0}\left(\omega_{x}-l \varphi\right)_{x t}+k l\left(\varphi_{x}+\psi+l \omega\right) \\
\quad+\gamma_{1} l\left(\varphi_{x}+\psi+l \omega\right)_{t}+\alpha \theta_{1 x}=0 \quad \text { in }(0, L) \times(0, \infty), \\
\theta_{1 t}-\theta_{1 x x}+\alpha\left(w_{x}-l \varphi\right)_{t}=0 \quad \text { in }(0, L) \times(0, \infty), \\
\theta_{2 t}-\theta_{2 x x}+\alpha \psi_{x t}=0 \quad \text { in }(0, L) \times(0, \infty) \\
\varphi(t, x)=\psi(t, x)=\omega(t, x)=\theta_{1}(t, x)=\theta_{2}(t, x)=0, \quad \text { for } \quad x=0, L, \\
\left(\varphi, \varphi_{t}, \psi, \psi_{t}, w, w_{t}, \theta_{1}, \theta_{2}\right)(x, 0)=\left(\varphi_{0}, \varphi_{1}, \psi, \psi_{1}, w_{0}, w_{1}, \theta_{10}, \theta_{20}\right)(x) .
\end{array}\right.
$$

with $t>0$ and $0<x<L$, where $L$ represents the distance between the ends of the center line of the beam, see figure. The functions $\varphi, \psi$ and $\omega$ denote, respectively, the transverse displacement of a curved beam, the rotation angle of the filament and the longitudinal displacement, while $\theta_{1}$ and $\theta_{2}$ are the temperature deviations along the longitudinal and vertical directions (see [10]). Here, $k_{0}=E H, k=G H$, $b=E I$ and $\rho_{1}, \rho_{2}, l, G, E, H$ and $\alpha$ denote positive constants characterizing physical properties of the beam and the filament. In addition, $l=\frac{1}{R}$, where $R$ is

Date: February 16, 2019.

Key words and phrases. Thermoviscoelastic Bresse beam; finite element discretization. 
the radius of curvature, and $\gamma_{0}, \gamma_{1}$ and $\gamma_{2}$ are viscosity coefficients. We denote by $u_{t}$ and $u_{x}$ the derivatives of $u$ with respect to $t$ and $x$.

The Bresse system is also known as the circular arch problem and is given by the following equations:

$$
\left\{\begin{array}{l}
\rho_{1} \varphi_{t t}=-\mathcal{Q}_{x}+l \mathcal{N} \\
\rho_{2} \psi_{t t}=\mathcal{M}_{x}+\mathcal{Q} \\
\rho_{1} \omega_{t t}=-\mathcal{N}_{x}-l \mathcal{Q} .
\end{array}\right.
$$

where

$$
\mathcal{N}=k_{0}\left(\omega_{x} l \varphi\right), \quad \mathcal{Q}=k\left(\varphi_{x}+\psi+l \omega\right), \quad \mathcal{M}=b \psi_{x}
$$

$\mathcal{N}, \mathcal{Q}$ and $\mathcal{M}$ to denote the axial force, the shear force and the bending moment.

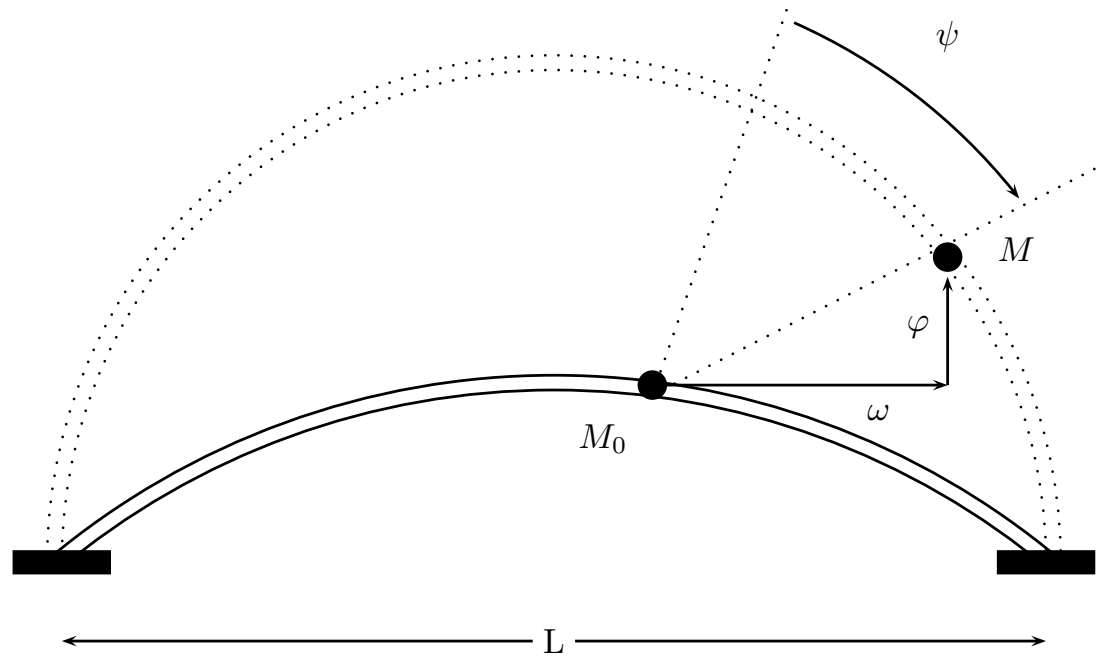

Figure: After deformation the particle $M_{0}$ of the beam take the position $M$

The energy of solutions of the system (1.1) is defined by

$$
\left\{\begin{aligned}
\mathcal{E}(t)=\frac{1}{2} \int_{0}^{L} & \left(\rho_{1}\left|\varphi_{t}\right|^{2}+\rho_{2}\left|\psi_{t}\right|^{2}+\rho_{1}\left|\omega_{t}\right|^{2}+b\left|\psi_{x}\right|^{2}\right. \\
& \left.+k\left|\varphi_{x}+\psi+l \omega\right|^{2}+k_{0}\left|\omega_{x}-l \varphi\right|^{2}+\theta_{1}^{2}+\theta_{2}^{2}\right) d x
\end{aligned}\right.
$$

This energy is decreasing. Indeed, a straightforward calculation gives

$$
\left\{\begin{array}{c}
\frac{d}{d t} \mathcal{E}(t)=-\int_{0}^{L}\left(\gamma_{1}\left|\left(\varphi_{x}+\psi+l \omega\right)_{t}\right|^{2}+\gamma_{2}\left|\psi_{x t}\right|^{2}+\gamma_{0}\left|\omega_{x t}-l \varphi_{t}\right|^{2}\right. \\
\left.+\left|\theta_{1 x}\right|^{2}+\left|\theta_{2 x}\right|^{2}\right) d x \leq 0
\end{array}\right.
$$

In the study of the thermoviscoelasticity of a beam, we take into account the effects of the viscoelastic and thermal dissipation energies upon the corresponding vibrations.

Many authors studied theoretically the stabilization of the thermoelastic Bresse beam and thermo(visco)elastic Timoshenko beam. In [12], Racke and Rivera considered the thermoelastic Timoshenko system and they established the exponential 
stability when $E=G$. Moreover, they also proved nonnexponential stability when $E \neq G$. In [10], Liu and Rao studied the energy decay rate for the thermoelastic Bresse system formed by three wave equations coupled with two heat equations. The two wave equations about the longitudinal displacement and shear angle displacement are damped by dissipation from the two heat equations. However, the wave equation about the vertical displacement is only weakly damped. The authors showed that, under conditions on the wave speeds, the energy decays exponentially. Recently, in [9], Gallego and Rivera studied the energy decay for the thermoelastic Bresse system in the whole line with two different dissipative mechanism, given by heat conduction (types I and III). They proved that the decay rate of the solutions are very slow.

In [4], Arwadi, Wehbeh and Youssef studied the observability of the elastic Bresse system in one-dimensional bounded domain. They introduced a new notion of observability which is the discrete observability by using an implicit Euler scheme in time and Lagrange finite elements in space.

On an other side, in [3], Copetti and Fernández considered the Timoshenko model for thick beam with viscosity and obtained some a priori error estimates for the numerical solution. In [1] Bernardi and Copetti considered a nonlinear model for a thermoviscoelastic Timoshenko beam that can enter in contact with obstacles. They proposed a discretization based on a combination of backward Euler and CrankNicolson schemes in time and finite elements in space and performed the a priori analysis of the discrete problem. They presented also some numerical experiments.

To our knowledge, this is the first paper to consider the thermoviscoelastic Bresse system (1.1) from the theoretical and numerical point of view. This system is a generalization of the thermoviscoelastic Timoshenko beam when $l$ and $w$ are neglected. The difference between this work and the others that study the Bresse system is the consideration of the viscoelasticity and thermoelasticity, which are physically important, and the performing of a priori analysis of a proposed discrete problem. In particular, exponential stability is derived without any restrictions on the wave velocities. Moreover, error estimates are obtained without using the standard technique which compares the solution of the discrete problem to some projection of the exact solution.

\section{WELL-POSEDNESS}

The energy space associated to problem (1.1) is defined by:

$$
\mathcal{H}=\left(H_{0}^{1}(0, L)\right)^{3} \times\left(L^{2}(0, L)\right)^{3} \times\left(H_{0}^{1}(0, L)\right)^{2} .
$$

The space $\mathcal{H}$ is equipped with the inner product that induces the energy norm. For $U=\left(\varphi, \psi, \omega, u, v, z, \theta_{1}, \theta_{2}\right) \in \mathcal{H}$, we define

$$
\left\{\begin{array}{c}
\|U\|_{\mathcal{H}}^{2}=\rho_{1}\|u\|^{2}+\rho_{2}\|v\|^{2}+\rho_{1}\|z\|^{2}+b\left\|\psi_{x}\right\|^{2}+k\left\|\varphi_{x}+\psi+l \omega\right\|^{2} \\
+k_{0}\left\|\omega_{x}-l \varphi\right\|^{2}+\left\|\theta_{1}\right\|^{2}+\left\|\theta_{2}\right\|^{2}
\end{array}\right.
$$

where $\|\cdot\|$ is the $L^{2}(0, L)$ norm. Using (3.1), we remark that the norm $\|U\|_{\mathcal{H}}$ is equivalent to the usual norm of $\mathcal{H}$ which is

$$
\|U\|^{2}=\left\|\varphi_{x}\right\|^{2}+\left\|\psi_{x}\right\|^{2}+\left\|\omega_{x}\right\|^{2}+\|u\|^{2}+\|v\|^{2}+\|z\|^{2}+\left\|\theta_{1}\right\|^{2}+\left\|\theta_{2}\right\|^{2} .
$$


Now, we introduce the linear unbounded operator $\mathcal{A}$ in $\mathcal{H}$ as follows:

$$
\mathcal{A} U=\left(\begin{array}{c}
u \\
v \\
z \\
\frac{k}{\rho_{1}}\left(\varphi_{x x}+\psi_{x}+l \omega_{x}\right)+\frac{l k_{0}}{\rho_{1}}\left(\omega_{x}-l \varphi\right)+\frac{\gamma_{1}}{\rho_{1}}\left(\varphi_{x}+\psi+l w\right)_{x t} \\
+\frac{k_{0}}{\rho_{1}} l\left(\omega_{x}-l \varphi\right)+\frac{l \gamma_{0}}{\rho_{1}}\left(w_{x}-l \varphi\right)_{t} \frac{l}{\rho_{1}} \alpha \theta_{1} \\
\frac{b}{\rho_{2}} \psi_{x x}-\frac{k}{\rho_{2}}\left(\varphi_{x}+\psi+l \omega\right)+\frac{\gamma_{2}}{\rho_{2}} \psi_{x x t}-\frac{k}{\rho_{2}}\left(\varphi_{x}+\psi+l \omega\right) \\
-\frac{\gamma_{1}}{\rho_{2}}\left(\varphi_{x}+l \omega+\psi\right)_{t}-\frac{\alpha}{\rho_{2}} \theta_{2 x} \\
\frac{k_{0}}{\rho_{1}}\left(\omega_{x x}-l \varphi_{x}\right)-\frac{l k}{\rho_{1}}\left(\varphi_{x}+\psi+l \omega\right)+\frac{\gamma_{0}}{\rho_{1}}\left(\omega_{x}-l \varphi\right)_{x t} \\
-\frac{l \gamma_{1}}{\rho_{1}}\left(\varphi_{x}+\psi+l \omega\right)_{t}-\frac{\alpha}{\rho_{1}} \theta_{1 x} \\
\theta_{1 x x}-\alpha\left(w_{x}-l \varphi\right)_{t} \\
\theta_{2 x x}-\alpha \psi_{x t}
\end{array}\right),
$$

$D(\mathcal{A})=\left\{\left(\varphi, \psi, \omega, u, v, z, \theta_{1}, \theta_{2}\right) \in \mathcal{H} ; \varphi, \psi, \omega, \theta_{1}, \theta_{2} \in H_{0}^{1}(0, L) \cap H^{2}(0, L), u, v, z \in H_{0}^{1}(0, L)\right\}$. Then, by substituting $u, v$ and $z$ by $u_{t} \cdot v_{t}$ and $z_{t}$ the thermoviscoelastic Bresse beam system can be written in the form of a first order evolution on the Hilbert space $\mathcal{H}$

$$
\left\{\begin{array}{l}
U^{\prime}=\mathcal{A} U \\
U(0)=U_{0}
\end{array}\right.
$$

Theorem 2.1. $\mathcal{A}$ generates a $\mathcal{C}_{0}$ semigroup $S(t)$ of contractions on $\mathcal{H}$.

Proof. Firstly, it is clear that $D(\mathcal{A})$ is dense in $\mathcal{H}$ and

$\langle\mathcal{A} U, U\rangle_{\mathcal{H}}=-\left(\gamma_{1}\left\|\left(\varphi_{x}+\psi+l \omega\right)_{t}\right\|^{2}+\gamma_{2}\left\|\psi_{x t}\right\|^{2}+\gamma_{0}\left\|\omega_{x t}-l \varphi_{t}\right\|^{2}+\left\|\theta_{1 x}\right\|^{2}+\left\|\theta_{2 x}\right\|^{2}\right) d x \leq 0$,

where $\langle., .\rangle_{\mathcal{H}}$ is the scalar product associated to the energy norm defined in (2.1). It remains to prove that

$$
\mathcal{A} U=f, \forall f \in D(\mathcal{A}),
$$

where $f=\left(f_{1}, f_{2}, \ldots, f_{8}\right)$, has a unique solution $U \in D(\mathcal{A})$. Indeed, the first three equations in (2.4) give

$$
u=f_{1}, \quad v=f_{2}, \quad \text { and } \quad z=f_{3} .
$$

Substituting $u, v$, and $z$ into the last two equation in (2.4) and using the standard elliptic PDE theory yield to the existence of the unique solution

$$
\theta_{1}, \theta_{2} \in H_{0}^{1} \cap H^{2} .
$$

On the other hand, if we consider the bilinear form defined by

$$
\begin{aligned}
& \mathbb{B}((\varphi, \psi, \omega),(\widehat{\varphi}), \widehat{\psi}, \widehat{\omega}) \\
& \quad:=\int_{0}^{L}\left[k\left(\varphi_{x}+\psi+l \omega\right)\left(\widehat{\varphi}_{x}+\widehat{\psi}+l \widehat{\omega}\right)+b \psi_{x} \widehat{\psi}_{x}+k_{0}\left(\omega_{x}-l \varphi\right)\left(\widehat{\omega}_{x}-l \widehat{\varphi}\right)\right] d x,
\end{aligned}
$$


and if we use the Lax-Milgram theorem we conclude that there exists a unique $(\varphi, \psi, \omega)$ solution of the fourth, fifth, and sixth equations of (2.4). Hence, $0 \in$ $\rho(\mathcal{A})$ and so by the resolvent identity, for small $\lambda>0$ we have $R(\lambda-\mathcal{A})=\mathcal{H}$. Consequently, the Lumer-Phillip theorem implies that $A$ generates a $\mathcal{C}_{0}$ semigroup $S(t)$ of contractions on $\mathcal{H}$. (For details see theorem 1.2.4 in [11])

The following existence and uniqueness result follows from the latter theorem.

Theorem 2.2. If $U_{0}=\left(\varphi_{0}, \psi_{0}, \omega_{0}, \varphi_{1}, \psi_{1}, \omega_{1}, \theta_{10}, \theta_{20}\right) \in D(\mathcal{A})$, problem (1.1) admits a unique classical solution $U=\left(\varphi, \psi, \omega, \varphi_{t}, \psi_{t}, \omega_{t}, \theta_{1}, \theta_{2}\right)$ such that

$$
U \in C([0,+\infty) ; D(\mathcal{A})) \cap C^{1}([0,+\infty) ; \mathcal{H}) .
$$

\section{Dissipation OF THE ENERGy}

In this section, we shall prove the exponential stabilization of the Bresse system which is based on the following inequality used in Haraux [6] and Lagnese [8] (see [7] for the proof).

Lemma 3.1. Let $E: \mathbb{R}_{+} \rightarrow \mathbb{R}_{+}$be a non-increasing function and assume that there exists a constant $T>0$ such that

$$
\int_{t}^{\infty} E(s) d s \leq T E(t), \quad \forall t \in \mathbb{R}_{+} .
$$

Then,

$$
E(t) \leq E(0) e^{1-\frac{t}{T}}, \quad \forall t \geq T .
$$

The next result will be needed in the proof of the exponential stabilization (see [13]).

Lemma 3.2. There exists a positive constant $C$ such that for every $(\varphi, \psi, \omega) \in$ $V:=\left(H_{0}^{1}(0, L)\right)^{3}$, we have

$$
\int_{0}^{L}\left(\left|\varphi_{x}\right|^{2}+\left|\psi_{x}\right|^{2}+\left|\omega_{x}\right|^{2}\right) d x \leq C \int_{0}^{L}\left(\left|\psi_{x}\right|^{2}+\left|\varphi_{x}+\psi+l \omega\right|^{2}+\left|\omega_{x}-l \varphi\right|^{2}\right) d x
$$

We obtain the same inequality if we replace the functions $\varphi, \psi$ and $\omega$ by $\varphi_{t}, \psi_{t}$ and $\omega_{t}$ i.e.

$\int_{0}^{L}\left(\left|\varphi_{x t}\right|^{2}+\left|\psi_{x t}\right|^{2}+\left|\omega_{x t}\right|^{2}\right) d x \leq C \int_{0}^{L}\left(\left|\psi_{x t}\right|^{2}+\left|\left(\varphi_{x}+\psi+l \omega\right)_{t}\right|^{2}+\left|\left(\omega_{x}-l \varphi\right)_{t}\right|^{2}\right) d x$.

Now, we are ready to show that the energy will decrease to zero exponentially when $t$ tends to $+\infty$.

Theorem 3.1. There exist two positive constants $C_{1}$ and $\eta$ such that the energy of the solution of (1.1) satisfies

$$
\mathcal{E}(t) \leq C_{1} \mathcal{E}(0) e^{-\eta t} \quad \forall t \geq 0 .
$$

Proof. Several steps are required for the proof of this theorem. For simplicity, we shall denote by $\int_{S}^{T} \int_{0}^{L} f d x d t=\int_{S}^{T} \int_{0}^{L} f$. 
Step 1. Multiplying the first equation of (1.1) by $\varphi$, the second by $\psi$, the third by $\omega$, the fourth by $\theta_{1}$, the fifth by $\theta_{2}$ and integrating over $[S, T] \times(0, L)$, we obtain:

$$
\left\{\begin{array}{l}
-\int_{S}^{T} \int_{0}^{L} \rho_{1}\left|\varphi_{t}\right|^{2}+\rho_{1} \int_{0}^{L}\left[\varphi_{t} \varphi\right]_{S}^{T} d x+k \int_{S}^{T} \int_{0}^{L}\left(\varphi_{x}+\psi+l \omega\right) \varphi_{x} \\
-\gamma_{1} \int_{S}^{T} \int_{0}^{L}\left(\varphi_{x}+\psi+l w\right)_{x t} \varphi-k_{0} l \int_{S}^{T} \int_{0}^{L}\left(\omega_{x}-l \varphi\right) \varphi \\
-l \gamma_{0} \int_{S}^{T} \int_{0}^{L}\left(w_{x}-l \varphi\right)_{t} \varphi+l \alpha \int_{S}^{T} \int_{0}^{L} \theta_{1} \varphi=0
\end{array}\right.
$$

$$
\begin{aligned}
& \left\{\begin{array}{l}
-\int_{S}^{T} \int_{0}^{L} \rho_{2}\left|\psi_{t}\right|^{2}+\rho_{2} \int_{0}^{L}\left[\psi_{t} \psi\right]_{S}^{T} d x+b \int_{S}^{T} \int_{0}^{L}\left|\psi_{x}\right|^{2} \\
+k \int_{S}^{T} \int_{0}^{L}\left(\varphi_{x}+\psi+l \omega\right) \psi-\gamma_{2} \int_{S}^{T} \int_{0}^{L} \psi_{x x t} \psi \\
+\gamma_{1} \int_{S}^{T} \int_{0}^{L}\left(\varphi_{x}+l \omega+\psi\right)_{t} \psi+\alpha \int_{S}^{T} \int_{0}^{L} \theta_{2 x} \psi=0
\end{array}\right. \\
& \left\{\begin{array}{l}
\int_{S}^{T} \int_{0}^{L} \rho_{1}\left|\omega_{t}\right|^{2}+\rho_{1} \int_{0}^{L}[\omega t \omega]_{S}^{T} d x+k_{0} \int_{S}^{T} \int_{0}^{L}\left(\omega_{x}-l \varphi\right) \omega_{x} \\
+l k \int_{S}^{T} \int_{0}^{L}\left(\varphi_{x}+\psi+l \omega\right) \omega-\gamma_{0} \int_{S}^{T} \int_{0}^{L}\left(\omega_{x}-l \varphi\right)_{x t} \omega \\
+\gamma_{1} l \int_{S}^{T} \int_{0}^{L}\left(\varphi_{x}+\psi+l \omega\right)_{t} \omega+\alpha \int_{S}^{T} \int_{0}^{L} \theta_{1 x} \omega=0 .
\end{array}\right.
\end{aligned}
$$

$$
\left\{\begin{array}{l}
\frac{1}{2} \int_{0}^{L}\left[\left|\theta_{1}(x, t)\right|^{2}\right]_{S}^{T} d x+\int_{S}^{T} \int_{0}^{L}\left|\theta_{1 x}\right|^{2} \\
+\alpha \int_{S}^{T} \int_{0}^{L}\left(w_{x}-l \varphi\right)_{t} \theta_{1}=0
\end{array}\right.
$$

and

$$
\left\{\begin{array}{l}
\frac{1}{2} \int_{0}^{L}\left[\left|\theta_{2}(x, t)\right|^{2}\right]_{S}^{T} d x+\int_{S}^{T} \int_{0}^{L}\left|\theta_{2 x}\right|^{2} \\
+\alpha \int_{S}^{T} \int_{0}^{L} \psi_{x t} \theta_{2}=0 .
\end{array}\right.
$$

For simplicity we shall denote by $I_{1}-$ 
7

Step 2. Adding (3.4), (3.5), (3.6), (3.7), and (3.8) leads to

$$
\left\{\begin{array}{l}
-\int_{S}^{T} \int_{0}^{L} \rho_{1}\left|\varphi_{t}\right|^{2}-\int_{S}^{T} \int_{0}^{L} \rho_{2}\left|\psi_{t}\right|^{2}-\int_{S}^{T} \int_{0}^{L} \rho_{1}\left|\omega_{t}\right|^{2} \\
+k \int_{S}^{T} \int_{0}^{L}\left|\varphi_{x}+\psi+l \omega\right|^{2}+k_{0} \int_{S}^{T} \int_{0}^{L}\left|\omega_{x}-l \varphi\right|^{2}+b \int_{S}^{T} \int_{0}^{L}\left|\psi_{x}\right|^{2} \\
+\rho_{1} \int_{0}^{L}\left[\varphi_{t} \varphi\right]_{S}^{T} d x+\rho_{2} \int_{0}^{L}\left[\psi_{t} \psi\right]_{S}^{T} d x+\rho_{1} \int_{0}^{L}\left[\omega_{t} \omega\right]_{S}^{T} d x \\
+\frac{\gamma_{1}}{2} \int_{S}^{T} \int_{0}^{L}\left(\left(\varphi_{x}+\psi+l \omega\right)^{2}\right)_{t}+\frac{\gamma_{2}}{2} \int_{S}^{T} \int_{0}^{L}\left(\psi_{x}^{2}\right)_{t} \\
+\frac{\gamma_{0}}{2} \int_{S}^{T} \int_{0}^{L}\left(\left(\omega_{x}-l \varphi\right)^{2}\right)_{t}+\frac{1}{2} \int_{0}^{L}\left[\left|\theta_{1}(x, t)\right|^{2}\right]_{S}^{T} d x+\int_{S}^{T} \int_{0}^{L}\left|\theta_{1 x}\right|^{2} \\
+\alpha \int_{S}^{T} \int_{0}^{L}\left(w_{x}-l \varphi\right)_{t} \theta_{1}+\frac{1}{2} \int_{0}^{L}\left[\left|\theta_{2}(x, t)\right|^{2}\right]_{S}^{T} d x+\int_{S}^{T} \int_{0}^{L}\left|\theta_{2 x}\right|^{2} \\
+\alpha \int_{S}^{T} \int_{0}^{L} \psi_{x t} \theta_{2}+l \alpha \int_{S}^{T} \int_{0}^{L} \theta_{1} \varphi+\alpha \int_{S}^{T} \int_{0}^{L} \theta_{2 x} \psi \\
+\alpha \int_{S}^{T} \int_{0}^{L} \theta_{1 x} \omega=0 .
\end{array}\right.
$$

From the definition of $\mathcal{E}$ we deduce

$$
\left\{\begin{aligned}
k \int_{0}^{L}\left|\varphi_{x}+\psi+l \omega\right|^{2} d x+k_{0} \int_{0}^{L}\left|\omega_{x}-l \varphi\right|^{2} d x+b \int_{0}^{L}\left|\psi_{x}\right|^{2} d x \\
=2 \mathcal{E}(t)-\int_{0}^{L} \rho_{1}\left|\varphi_{t}\right|^{2} d x-\int_{0}^{L} \rho_{2}\left|\psi_{t}\right|^{2} d x-\int_{0}^{L} \rho_{1}\left|\omega_{t}\right|^{2} d x \\
\quad-\int_{0}^{L}\left|\theta_{1}\right|^{2} d x-\int_{0}^{L}\left|\theta_{2}\right|^{2} d x
\end{aligned}\right.
$$

By combining (3.9) and (3.10) we get (3.11)

$$
\left\{\begin{array}{l}
2 \int_{S}^{T} \mathcal{E}(t) d t=-\rho_{1} \int_{0}^{L}\left[\varphi_{t} \varphi\right]_{S}^{T} d x+\rho_{2} \int_{0}^{L}\left[\psi_{t} \psi\right]_{S}^{T} d x+\rho_{1} \int_{0}^{L}\left[\omega_{t} \omega\right]_{S}^{T} d x \\
+2\left(\int_{S}^{T} \int_{0}^{L} \rho_{1}\left|\varphi_{t}\right|^{2}+\int_{S}^{T} \int_{0}^{L} \rho_{2}\left|\psi_{t}\right|^{2}+\int_{S}^{T} \int_{0}^{L} \rho_{1}\left|\omega_{t}\right|^{2}\right) \\
+\int_{S}^{T} \int_{0}^{L}\left|\theta_{1}\right|^{2}+\int_{S}^{T} \int_{0}^{L}\left|\theta_{2}\right|^{2}-\int_{S}^{T} \int_{0}^{L}\left|\theta_{1 x}\right|^{2}-\int_{S}^{T} \int_{0}^{L}\left|\theta_{2 x}\right|^{2} \\
-\frac{\gamma_{1}}{2} \int_{S}^{T} \int_{0}^{L}\left(\left(\varphi_{x}+\psi+l \omega\right)^{2}\right)_{t}-\frac{\gamma_{2}}{2} \int_{S}^{T} \int_{0}^{L}\left(\psi_{x}^{2}\right)_{t} \\
-\frac{\gamma_{0}}{2} \int_{S}^{T} \int_{0}^{L}\left(\left(\omega_{x}-l \varphi\right)^{2}\right)_{t}-\frac{1}{2} \int_{0}^{L}\left[\left|\theta_{1}(x, t)\right|^{2}\right]_{S}^{T} d x-\frac{1}{2} \int_{0}^{L}\left[\left|\theta_{2}(x, t)\right|^{2}\right]_{S}^{T} d x \\
-\alpha \int_{S}^{T} \int_{0}^{L}\left(w_{x}-l \varphi\right)_{t} \theta_{1}-\alpha \int_{S}^{T} \int_{0}^{L} \psi_{x t} \theta_{2} \\
-l \alpha \int_{S}^{T} \int_{0}^{L} \theta_{1} \varphi-\alpha \int_{S}^{T} \int_{0}^{L} \theta_{2 x} \psi-\alpha \int_{S}^{T} \int_{0}^{L} \theta_{1 x} \omega .
\end{array}\right.
$$


The first term $-\frac{\gamma_{1}}{2} \int_{S}^{T} \int_{0}^{L}\left(\left(\varphi_{x}+\psi+l \omega\right)^{2}\right)_{t}$ is given by

$$
\begin{aligned}
& -\frac{\gamma_{1}}{2} \int_{S}^{T} \int_{0}^{L}\left(\left(\varphi_{x}+\psi+l \omega\right)^{2}\right)_{t} \\
& \quad=-\frac{\gamma_{1}}{2} \int_{0}^{L}\left[\left(\varphi_{x}+\psi+l \omega\right)^{2}\right]_{S}^{T} d x \\
& \leq \frac{\gamma_{1}}{2} \int_{0}^{L}\left[\left(\varphi_{x}+\psi+l \omega\right)^{2}\right]_{S}^{T} \\
& \quad \leq \frac{\gamma_{1}}{2} \int_{0}^{L}\left[\left(\varphi_{x}+\psi+l \omega\right)^{2}(T)\right. \\
& \leq \frac{\gamma_{1}}{k} \mathcal{E}(T) \quad(\text { by the definition of } \mathcal{E}) \\
& \quad \leq \frac{\gamma_{1}}{k} \mathcal{E}(S) \quad(\text { since } \mathcal{E} \text { is decreasing }) .
\end{aligned}
$$

Hence,

$$
-\frac{\gamma_{1}}{2} \int_{S}^{T} \int_{0}^{L}\left(\left(\varphi_{x}+\psi+l \omega\right)^{2}\right)_{t} \leq \frac{\gamma_{1}}{k} \mathcal{E}(S)
$$

Similarly, we have

$$
-\frac{\gamma_{2}}{2} \int_{S}^{T} \int_{0}^{L}\left(\psi_{x}^{2}\right)_{t} \leq \frac{\gamma_{2}}{b} \mathcal{E}(S)
$$

and

$$
-\frac{\gamma_{0}}{2} \int_{S}^{T} \int_{0}^{L}\left(\left(\omega_{x}-l \varphi\right)^{2}\right)_{t} \leq \frac{\gamma_{0}}{k_{0}} \mathcal{E}(S) .
$$

Therefore, using (3.12), (3.13), and (3.14) in (3.11) yields (3.15)

$$
\left\{\begin{array}{l}
2 \int_{S}^{T} \mathcal{E}(t) d t \leq-\rho_{1} \int_{0}^{L}\left[\varphi_{t} \varphi\right]_{S}^{T} d x+\rho_{2} \int_{0}^{L}\left[\psi_{t} \psi\right]_{S}^{T} d x+\rho_{1} \int_{0}^{L}\left[\omega_{t} \omega\right]_{S}^{T} d x \\
+2\left(\int_{S}^{T} \int_{0}^{L} \rho_{1}\left|\varphi_{t}\right|^{2}+\int_{S}^{T} \int_{0}^{L} \rho_{2}\left|\psi_{t}\right|^{2}+\int_{S}^{T} \int_{0}^{L} \rho_{1}\left|\omega_{t}\right|^{2}\right) \\
+\int_{S}^{T} \int_{0}^{L}\left|\theta_{1}\right|^{2}+\int_{S}^{T} \int_{0}^{L}\left|\theta_{2}\right|^{2}-\frac{1}{2} \int_{0}^{L}\left[\left|\theta_{1}(x, t)\right|^{2}\right]_{S}^{T} d x-\frac{1}{2} \int_{0}^{L}\left[\left|\theta_{2}(x, t)\right|^{2}\right]_{S}^{T} d x \\
-\alpha \int_{S}^{T} \int_{0}^{L}\left(w_{x}-l \varphi\right)_{t} \theta_{1}-\alpha \int_{S}^{T} \int_{0}^{L} \psi_{x t} \theta_{2} \\
-l \alpha \int_{S}^{T} \int_{0}^{L} \theta_{1} \varphi-\alpha \int_{S}^{T} \int_{0}^{L} \theta_{2 x} \psi-\alpha \int_{S}^{T} \int_{0}^{L} \theta_{1 x} \omega \\
+\left(\frac{\gamma_{0}}{k_{0}}+\frac{\gamma_{1}}{k}+\frac{\gamma_{2}}{b}\right) \mathcal{E}(S) \forall S \in(0, T) .
\end{array}\right.
$$

Step 3. In this step, we shall estimate the terms of the right member of (3.15). By using the Young inequality, the Poincaré inequality, and the estimation (3.1) we deduce

$$
-\rho_{1} \int_{0}^{L}\left[\varphi_{t} \varphi\right]_{S}^{T} d x \leq c \mathcal{E}(S) \forall S \in(0, T)
$$




$$
-\rho_{2} \int_{0}^{L}\left[\psi_{t} \psi\right]_{S}^{T} d x \leq c \mathcal{E}(S) \forall S \in(0, T)
$$

and

$$
-\rho_{1} \int_{0}^{L}\left[\omega_{t} \omega\right]_{S}^{T} d x \leq c \mathcal{E}(S) \forall S \in(0, T),
$$

where $c$ is a generic constant that depends on $\rho_{1}, \rho_{2}, k_{0}, k, b$, and the Poincaré constant.

On the other hand, the Poincare inequality and the relation (3.2) lead to

$$
\begin{aligned}
& 2\left(\int_{S}^{T} \int_{0}^{L}\left(\rho_{1}\left|\varphi_{t}\right|^{2}+\rho_{2}\left|\psi_{t}\right|^{2}+\rho_{1}\left|\omega_{t}\right|^{2}\right)\right) \\
& \quad \leq c\left(\int_{S^{T}}^{T} \int_{0}^{L}\left(\left|\varphi_{x t}\right|^{2}+\left|\psi_{x t}\right|^{2}+\left|\omega_{x t}\right|^{2}\right)\right) \\
& \quad \leq c\left(\int_{S}^{T} \int_{0}^{L}\left(\left|\left(\varphi_{x}+\psi+l \omega\right)_{t}\right|^{2}+\left|\psi_{x t}\right|^{2}+\left|\left(\omega_{x}-l \varphi\right)_{t}\right|^{2}\right)\right) \leq c \mathcal{E}(S) \forall S \in(0, T) .
\end{aligned}
$$

But,

$$
\begin{aligned}
& \left(\int_{S}^{T} \int_{0}^{L}\left(\left|\left(\varphi_{x}+\psi+l \omega\right)_{t}\right|^{2}+\left|\psi_{x t}\right|^{2}+\left|\left(\omega_{x}-l \varphi\right)_{t}\right|^{2}\right)\right) \\
& \quad \leq c \int_{S}^{T}\left(-\mathcal{E}^{\prime}\right) d t \leq c \mathcal{E}(S) \quad \forall S \in(0, T) .
\end{aligned}
$$

Therefore,

$$
2\left(\int_{S}^{T} \int_{0}^{L}\left(\rho_{1}\left|\varphi_{t}\right|^{2}+\rho_{2}\left|\psi_{t}\right|^{2}+\rho_{1}\left|\omega_{t}\right|^{2}\right)\right) \leq c \mathcal{E}(S) \quad \forall S \in(0, T) .
$$

On the other hand, from the Poincaré inequality, easily we conclude the following estimation

$$
\left\{\begin{array}{l}
\int_{S}^{T} \int_{0}^{L}\left|\theta_{1}\right|^{2}+\int_{S}^{T} \int_{0}^{L}\left|\theta_{2}\right|^{2} \leq c\left(\int_{S}^{T} \int_{0}^{L}\left|\theta_{1 x}\right|^{2}+\int_{S}^{T} \int_{0}^{L}\left|\theta_{2 x}\right|^{2}\right) \\
c \int_{S}^{T}\left(-\mathcal{E}^{\prime}\right) d t \leq c \mathcal{E}(S) \quad \forall S \in(0, T) .
\end{array}\right.
$$

Now, the Cauchy-Schwarz inequality gives us

$$
\int_{S}^{T} \int_{0}^{L}\left(w_{x}-l \varphi\right)_{t} \theta_{1} \leq \int_{S}^{T}\left(\int_{0}^{L}\left|\left(w_{x}-l \varphi\right)_{t}\right|^{2} d x\right)^{\frac{1}{2}}\left(\int_{0}^{L}\left|\theta_{1}\right|^{2} d x\right)^{\frac{1}{2}} d t .
$$

But, from (1.4) and the definition of $\mathcal{E}$, we have

$$
\int_{0}^{L}\left|\left(w_{x}-l \varphi\right)_{t}\right|^{2} d x \leq c \int_{S}^{T}\left(-\mathcal{E}^{\prime}\right) d t, \quad \int_{0}^{L}\left|\theta_{1}\right|^{2} d x \leq 2 \mathcal{E}(t) \forall t>0 .
$$

Hence, (3.21) implies

$$
\int_{S}^{T} \int_{0}^{L}\left(w_{x}-l \varphi\right)_{t} \theta_{1} \leq c \int_{S}^{T}\left(-\mathcal{E}^{\prime}\right)^{\frac{1}{2}} \mathcal{E}^{\frac{1}{2}} d t .
$$

Now, for all $\varepsilon>0$, Young's inequality gives

$$
\int_{S}^{T} \int_{0}^{L}\left(w_{x}-l \varphi\right)_{t} \theta_{1} \leq \frac{c}{\varepsilon} \int_{S}^{T}\left(-\mathcal{E}^{\prime}\right) d t+\varepsilon \int_{S}^{T} \mathcal{E} d t
$$


and so

$$
\int_{S}^{T} \int_{0}^{L}\left(w_{x}-l \varphi\right)_{t} \theta_{1} \leq c \mathcal{E}(S)+\varepsilon \int_{S}^{T} \mathcal{E} d t \quad \forall S \in(0, T)
$$

Repeating the same argument to $\int_{S}^{T} \int_{0}^{L} \psi_{x t} \theta_{2}$, we conclude that

$$
\int_{S}^{T} \int_{0}^{L} \psi_{x t} \theta_{2} \leq c \mathcal{E}(S)+\varepsilon \int_{S}^{T} \mathcal{E}(t) d t \quad \forall S \in(0, T)
$$

Concerning the term $-\frac{1}{2} \int_{0}^{L}\left[\left|\theta_{1}\right|^{2}\right]_{S}^{T} d x$, we have:

$-\frac{1}{2} \int_{0}^{L}\left[\left|\theta_{1}\right|^{2}\right]_{S}^{T} d x=\frac{1}{2} \int_{0}^{L}\left|\theta_{1}(S)\right|^{2} d x-\frac{1}{2} \int_{0}^{L}\left|\theta_{1}(T)\right|^{2} d x \leq \frac{1}{2} \int_{0}^{L}\left|\theta_{1}(T)\right|^{2} d x \leq \mathcal{E}(T) \leq \mathcal{E}(S)$,

since $\mathcal{E}$ is decreasing. Therefore,

$$
-\frac{1}{2} \int_{0}^{L}\left[\left|\theta_{1}\right|^{2}\right]_{S}^{T} d x \leq \mathcal{E}(S)
$$

and by the same calculation we have

$$
-\frac{1}{2} \int_{0}^{L}\left[\left|\theta_{2}\right|^{2}\right]_{S}^{T} d x \leq \mathcal{E}(S)
$$

The estimation of the terms in the fifth row of (3.15) can be obtained by using Poincaré's inequality, (3.1), and (1.4). So,

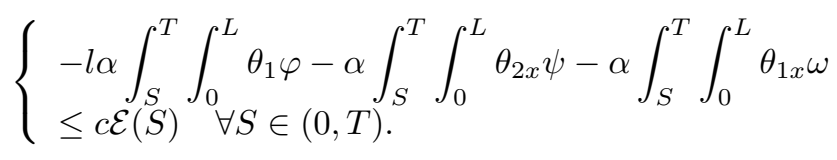

Step 4. Using (3.16), (3.17), (3.18), (3.19), (3.20), (3.22), (3.23), (3.24), (3.25), and $(3.26)$ in $(3.15)$ yields

$$
2 \int_{S}^{T} \mathcal{E}(t) d t \leq c \mathcal{E}(S)+\varepsilon \int_{S}^{T} \mathcal{E}(t) d t \forall S \in(0, T)
$$

and so

$$
\int_{S}^{T} \mathcal{E}(t) d t \leq c \mathcal{E}(S), \forall S \in(0, T) .
$$

Thus, if we fix $S$ and make $T \longrightarrow+\infty$, the proof is complete by applying lemma 3.1 .

\section{Numerical approximation}

In this section, we propose a numerical approximation to the solution of the continuous problem (1.1). Let us introduce the functions $\tilde{\varphi}=\varphi_{t}, \tilde{\psi}=\psi_{t}, \tilde{\omega}=\omega_{t}$ 
and the weak form associated to system (1.1) obtained by multiplying the equations by test functions $\zeta, \chi, \xi, \eta_{1}, \eta_{2} \in H_{0}^{1}(0, L)$ and integrating by parts:

$$
\left\{\begin{array}{c}
\rho_{1}\left(\tilde{\varphi}_{t}, \zeta\right)+k\left(\varphi_{x}+\psi+l \omega, \zeta_{x}\right)+\gamma_{1}\left(\tilde{\varphi}_{x}+\tilde{\psi}+l \tilde{\omega}, \zeta_{x}\right)-k_{0} l\left(\omega_{x}-l \varphi, \zeta\right) \\
-\gamma_{0} l\left(\tilde{\omega}_{x}-l \tilde{\varphi}, \zeta\right)+l \alpha\left(\theta_{1}, \zeta\right)=0 \\
\rho_{2}\left(\tilde{\psi}_{t}, \chi\right)+b\left(\psi_{x}, \chi_{x}\right)+\gamma_{2}\left(\tilde{\psi}_{x}, \chi_{x}\right)+k\left(\varphi_{x}+\psi+l \omega, \chi\right) \\
+\gamma_{1}\left(\tilde{\varphi}_{x}+\tilde{\psi}+l \tilde{\omega}, \chi\right)-\alpha\left(\theta_{2}, \chi_{x}\right)=0 \\
\rho_{1}\left(\tilde{\omega}_{t}, \xi\right)+k_{0}\left(\omega_{x}-l \varphi, \xi_{x}\right)+\gamma_{0}\left(\tilde{\omega}_{x}-l \tilde{\varphi}, \xi_{x}\right)+k l\left(\varphi_{x}+\psi+l \omega, \xi\right) \\
+\gamma_{1} l\left(\tilde{\varphi}_{x}+\tilde{\psi}+l \tilde{\omega}, \xi\right)-\alpha\left(\theta_{1}, \xi_{x}\right)=0 \\
\left(\theta_{1 t}, \eta_{1}\right)+\left(\theta_{1 x}, \eta_{1 x}\right)+\alpha\left(\tilde{\omega}_{x}-l \tilde{\varphi}, \eta_{1}\right)=0 \\
\left(\theta_{2 t}, \eta_{2}\right)+\left(\theta_{2 x}, \eta_{2 x}\right)+\alpha\left(\tilde{\psi}_{x}, \eta_{2}\right)=0
\end{array}\right.
$$

Partition the interval $\Omega$ into subintervals $I_{j}=\left(x_{j-1}, x_{j}\right)$ of length $h=L / s$, with $0=x_{0}<x_{1}<\cdots<x_{s}=L$, and denote by $S_{h} \subset H_{0}^{1}(0, L)$ the space of continuous piecewise linear functions defined on this partition. For a given final time $T>0$ and a positive integer $N$, let $\Delta t=T / N$ be the time step.

The finite element method using the backward Euler scheme is to find $\tilde{\varphi}_{h}^{n}, \tilde{\psi}_{h}^{n}, \tilde{\omega}_{h}^{n}$, $\theta_{1 h}^{n}, \theta_{2 h}^{n} \in S_{h}$ such that, for $n=1, \ldots, N$ and for all $\zeta_{h}, \chi_{h}, \xi_{h}, \eta_{1 h}, \eta_{2 h} \in S_{h}$

$$
\left\{\begin{array}{c}
\frac{\rho_{1}}{\Delta t}\left(\tilde{\varphi}_{h}^{n}-\tilde{\varphi}_{h}^{n-1}, \zeta_{h}\right)+k\left(\varphi_{h x}^{n}+\psi_{h}^{n}+l \omega_{h}^{n}, \zeta_{h x}\right) \\
\quad+\gamma_{1}\left(\tilde{\varphi}_{h x}^{n}+\tilde{\psi}_{h}^{n}+l \tilde{\omega}_{h}^{n}, \zeta_{h x}\right)-k_{0} l\left(\omega_{h x}^{n}-l \varphi_{h}^{n}, \zeta_{h}\right) \\
\quad-\gamma_{0} l\left(\tilde{\omega}_{h x}^{n}-l \tilde{\varphi}_{h}^{n}, \zeta_{h}\right)+l \alpha\left(\theta_{1 h}^{n}, \zeta_{h}\right)=0, \\
\frac{\rho_{2}}{\Delta t}\left(\tilde{\psi}_{h}^{n}-\tilde{\psi}_{h}^{n-1}, \chi_{h}\right)+b\left(\psi_{h x}^{n}, \chi_{h x}\right)+\gamma_{2}\left(\tilde{\psi}_{h x}^{n}, \chi_{h x}\right) \\
\quad+k\left(\varphi_{h x}^{n}+\psi_{h}^{n}+l \omega_{h}^{n}, \chi_{h}\right)+\gamma_{1}\left(\tilde{\varphi}_{h x}^{n}+\tilde{\psi}_{h}^{n}+l \tilde{\omega}_{h}^{n}, \chi_{h}\right) \\
\quad-\alpha\left(\theta_{2 h}^{n}, \chi_{h x}\right)=0 \\
\frac{\rho_{1}}{\Delta t}\left(\tilde{\omega}_{h}^{n}-\tilde{\omega}_{h}^{n-1}, \xi_{h}\right)+k_{0}\left(\omega_{h x}^{n}-l \varphi_{h}^{n}, \xi_{h x}\right)+\gamma_{0}\left(\tilde{\omega}_{h x}^{n}-l \tilde{\varphi}_{h}^{n}, \xi_{h x}\right) \\
\quad+k l\left(\varphi_{h x}^{n}+\psi_{h}^{n}+l \omega_{h}^{n}, \xi_{h}\right)+\gamma_{1} l\left(\tilde{\varphi}_{h x}^{n}+\tilde{\psi}_{h}^{n}+l \tilde{\omega}_{h}^{n}, \xi_{h}\right) \\
\quad-\alpha\left(\theta_{1 h}^{n}, \xi_{h x}\right)=0, \\
\frac{1}{\Delta t}\left(\theta_{1 h}^{n}-\theta_{1 h}^{n-1}, \eta_{1 h}\right)+\left(\theta_{1 h x}^{n}, \eta_{1 h x}\right)+\alpha\left(\tilde{\omega}_{h x}^{n}-l \tilde{\varphi}_{h}^{n}, \eta_{1 h}\right)=0, \\
\frac{1}{\Delta t}\left(\theta_{2 h}^{n}-\theta_{2 h}^{n-1}, \eta_{2 h}\right)+\left(\theta_{2 h x}^{n}, \eta_{2 h x}\right)+\alpha\left(\tilde{\psi}_{h x}^{n}, \eta_{2 h}\right)=0,
\end{array}\right.
$$

where

$$
\tilde{\varphi}_{h}^{n}=\frac{\varphi_{h}^{n}-\varphi_{h}^{n-1}}{\Delta t}, \quad \tilde{\psi}_{h}^{n}=\frac{\psi_{h}^{n}-\psi_{h}^{n-1}}{\Delta t}, \quad \tilde{\omega}_{h}^{n}=\frac{\omega_{h}^{n}-\omega_{h}^{n-1}}{\Delta t}
$$

are approximations to $\varphi_{t}\left(t_{n}\right), \psi_{t}\left(t_{n}\right)$ and $\omega_{t}\left(t_{n}\right)$, respectively. Here, $\varphi_{h}^{0}, \tilde{\varphi}_{h}^{0}, \psi_{h}^{0}$, $\tilde{\psi}_{h}^{0}, \omega_{h}^{0}, \tilde{\omega}_{h}^{0}, \theta_{1 h}^{0}, \theta_{2 h}^{0}$ are given approximations to the initial conditions $\varphi_{0}, \varphi_{1}, \psi_{0}$, $\psi_{1}, \omega_{0}, \omega_{1}, \theta_{10}, \theta_{20}$, respectively.

The following equality will often be used:

$$
(a-b, a)=\frac{1}{2}\left(\|a-b\|^{2}+\|a\|^{2}-\|b\|^{2}\right)
$$


The next result is a discrete version of the energy decay property satisfied by the solution of system (1.1)

Theorem 4.1. Let the discrete energy be

$$
\begin{aligned}
\mathcal{E}_{h}^{n}= & \frac{1}{2}\left(\rho_{1}\left(\left\|\tilde{\varphi}_{h}^{n}\right\|^{2}+\left\|\tilde{\omega}_{h}^{n}\right\|^{2}\right)+\rho_{2}\left\|\tilde{\psi}_{h}^{n}\right\|^{2}+b\left\|\psi_{h x}^{n}\right\|^{2}+k\left\|\varphi_{h x}^{n}+\psi_{h}^{n}+l \omega_{h}^{n}\right\|^{2}\right. \\
& \left.+k_{0}\left\|\omega_{h x}^{n}-l \varphi_{h}^{n}\right\|^{2}+\left\|\theta_{1 h}^{n}\right\|^{2}+\left\|\theta_{2 h}^{n}\right\|^{2}\right) .
\end{aligned}
$$

Then, the decay property

$$
\frac{\mathcal{E}_{h}^{n}-\mathcal{E}_{h}^{n-1}}{\Delta t} \leq 0
$$

holds for $n=1,2, \ldots, N$.

Proof. Taking $\zeta_{h}=\tilde{\varphi}_{h}^{n}, \chi_{h}=\tilde{\psi}_{h}^{n}, \xi_{h}=\tilde{\omega}_{h}^{n}, \eta_{1 h}=\theta_{1 h}^{n}$ and $\eta_{2 h}=\theta_{2 h}^{n}$ it results that

$$
\left\{\begin{array}{c}
\frac{\rho_{1}}{2 \Delta t}\left(\left\|\tilde{\varphi}_{h}^{n}-\tilde{\varphi}_{h}^{n-1}\right\|^{2}+\left\|\tilde{\varphi}_{h}^{n}\right\|^{2}-\left\|\tilde{\varphi}_{h}^{n-1}\right\|^{2}\right)+k\left(\varphi_{h x}^{n}+\psi_{h}^{n}+l \omega_{h}^{n}, \tilde{\varphi}_{h x}^{n}\right) \\
+\gamma_{1}\left(\tilde{\varphi}_{h x}^{n}+\tilde{\psi}_{h}^{n}+l \tilde{\omega}_{h}^{n}, \tilde{\varphi}_{h x}^{n}\right)-k_{0} l\left(\omega_{h x}^{n}-l \varphi_{h}^{n}, \tilde{\varphi}_{h}^{n}\right) \\
-\gamma_{0} l\left(\tilde{\omega}_{h x}^{n}-l \tilde{\varphi}_{h}^{n}, \tilde{\varphi}_{h}^{n}\right)+l \alpha\left(\theta_{1 h}^{n}, \tilde{\varphi}_{h}^{n}\right)=0
\end{array}\right.
$$

$$
\left\{\begin{array}{c}
\frac{\rho_{2}}{2 \Delta t}\left(\left\|\tilde{\psi}_{h}^{n}-\tilde{\psi}_{h}^{n-1}\right\|^{2}+\left\|\tilde{\psi}_{h}^{n}\right\|^{2}-\left\|\tilde{\psi}_{h}^{n-1}\right\|^{2}\right)+k\left(\varphi_{h x}^{n}+\psi_{h}^{n}+l \omega_{h}^{n}, \tilde{\psi}_{h}^{n}\right) \\
+\frac{b}{2 \Delta t}\left(\left\|\psi_{h x}^{n}-\psi_{h x}^{n-1}\right\|^{2}+\left\|\psi_{h x}^{n}\right\|^{2}-\left\|\psi_{h x}^{n-1}\right\|^{2}\right)+\gamma_{2}\left\|\tilde{\psi}_{h x}^{n}\right\|^{2} \\
+\gamma_{1}\left(\tilde{\varphi}_{h x}^{n}+\tilde{\psi}_{h}^{n}+l \tilde{\omega}_{h}^{n}, \tilde{\psi}_{h}^{n}\right)-\alpha\left(\theta_{2 h}^{n}, \tilde{\psi}_{h x}^{n}\right)=0
\end{array}\right.
$$

$$
\left\{\begin{array}{c}
\frac{\rho_{1}}{2 \Delta t}\left(\left\|\tilde{\omega}_{h}^{n}-\tilde{\omega}_{h}^{n-1}\right\|^{2}+\left\|\tilde{\omega}_{h}^{n}\right\|^{2}-\left\|\tilde{\omega}_{h}^{n-1}\right\|^{2}\right)+k l\left(\varphi_{h x}^{n}+\psi_{h}^{n}+l \omega_{h}^{n}, \tilde{\omega}_{h}^{n}\right) \\
+\gamma_{1} l\left(\tilde{\varphi}_{h x}^{n}+\tilde{\psi}_{h}^{n}+l \tilde{\omega}_{h}^{n}, \tilde{\omega}_{h}^{n}\right)+k_{0}\left(\omega_{h x}^{n}-l \varphi_{h}^{n}, \tilde{\omega}_{h x}^{n}\right) \\
+\gamma_{0}\left(\tilde{\omega}_{h x}^{n}-l \tilde{\varphi}_{h}^{n}, \tilde{\omega}_{h x}^{n}\right)-\alpha\left(\theta_{1 h}^{n}, \tilde{\omega}_{h x}^{n}\right)=0
\end{array}\right.
$$

$$
\left\{\begin{array}{c}
\frac{1}{2 \Delta t}\left(\left\|\theta_{1 h}^{n}-\theta_{1 h}^{n-1}\right\|^{2}+\left\|\theta_{1 h}^{n}\right\|^{2}-\left\|\theta_{1 h}^{n-1}\right\|^{2}\right)+\left\|\theta_{1 h x}^{n}\right\|^{2} \\
+\alpha\left(\tilde{\omega}_{h x}^{n}-l \tilde{\varphi}_{h}^{n}, \theta_{1 h}^{n}\right)=0
\end{array}\right.
$$

and

$$
\left\{\begin{array}{c}
\frac{1}{2 \Delta t}\left(\left\|\theta_{2 h}^{n}-\theta_{2 h}^{n-1}\right\|^{2}+\left\|\theta_{2 h}^{n}\right\|^{2}-\left\|\theta_{2 h}^{n-1}\right\|^{2}\right)+\left\|\theta_{2 h x}^{n}\right\|^{2} \\
+\alpha\left(\tilde{\psi}_{h x}^{n}, \theta_{2 h}^{n}\right)=0 .
\end{array}\right.
$$

Recalling (4.3) and (4.4) we deduce that

$$
\begin{aligned}
& k\left(\varphi_{h x}^{n}+\psi_{h}^{n}+l \omega_{h}^{n}, \tilde{\varphi}_{h x}^{n}+\tilde{\psi}_{h}^{n}+l \tilde{\omega}_{h}^{n}\right) \\
& \quad \geq \frac{k}{2 \Delta t}\left(\left\|\varphi_{h x}^{n}+\psi_{h}^{n}+l \omega_{h}^{n}\right\|^{2}-\left\|\varphi_{h x}^{n-1}+\psi_{h}^{n-1}+l \omega_{h}^{n-1}\right\|^{2}\right) .
\end{aligned}
$$

and that

$$
k_{0}\left(\omega_{h x}^{n}-l \varphi_{h}^{n}, \tilde{\omega}_{h x}^{n}-l \tilde{\varphi}_{h}^{n}\right) \geq \frac{k_{0}}{2 \Delta t}\left(\left\|\omega_{h x}^{n}-l \varphi_{h}^{n}\right\|^{2}-\left\|\omega_{h x}^{n-1}-l \varphi_{h}^{n-1}\right\|^{2}\right) .
$$


Thus, after summing equations (4.5)-(4.9), we arrive at

$$
\begin{aligned}
& \frac{\rho_{1}}{2 \Delta t}\left(\left\|\tilde{\varphi}_{h}^{n}\right\|^{2}-\left\|\tilde{\varphi}_{h}^{n-1}\right\|^{2}+\left\|\tilde{\omega}_{h}^{n}\right\|^{2}-\left\|\tilde{\omega}_{h}^{n-1}\right\|^{2}\right)+\frac{\rho_{2}}{2 \Delta t}\left(\left\|\tilde{\psi}_{h}^{n}\right\|^{2}-\left\|\tilde{\psi}_{h}^{n-1}\right\|^{2}\right) \\
& \quad+\frac{b}{2 \Delta t}\left(\left\|\psi_{h x}^{n}\right\|^{2}-\left\|\psi_{h x}^{n-1}\right\|^{2}\right)+\gamma_{2}\left\|\tilde{\psi}_{h x}^{n}\right\|^{2} \\
& \quad+\frac{1}{2 \Delta t}\left(\left\|\theta_{1 h}^{n}\right\|^{2}-\left\|\theta_{1 h}^{n-1}\right\|^{2}+\left\|\theta_{2 h}^{n}\right\|^{2}-\left\|\theta_{2 h}^{n-1}\right\|^{2}\right)+\left\|\theta_{1 h x}^{n}\right\|^{2}+\left\|\theta_{2 h x}^{n}\right\|^{2} \\
& \quad+\frac{k}{2 \Delta t}\left(\left\|\varphi_{h x}^{n}+\psi_{h}^{n}+l \omega_{h}^{n}\right\|^{2}-\left\|\varphi_{h x}^{n-1}+\psi_{h}^{n-1}+l \omega_{h}^{n-1}\right\|^{2}\right) \\
& \quad+\frac{k_{0}}{2 \Delta t}\left(\left\|\omega_{h x}^{n}-l \varphi_{h}^{n}\right\|^{2}-\left\|\omega_{h x}^{n-1}-l \varphi_{h}^{n-1}\right\|^{2}\right) \\
& \quad+\gamma_{1}\left\|\tilde{\varphi}_{h x}^{n}+\tilde{\psi}_{h}^{n}+l \tilde{\omega}_{h}^{n}\right\|^{2}+\gamma_{0}\left\|\tilde{\omega}_{h x}^{n}-l \tilde{\varphi}_{h}^{n}\right\|^{2} \leq 0 .
\end{aligned}
$$

which proves the result.

Remark 4.1. Note that to find the solution of (4.2) a square linear system of algebraic equations needs to be solved. It follows from the above proof that when all data are zero, the solution $\left\{\tilde{\varphi}_{h}^{n}, \tilde{\psi}_{h}^{n}, \tilde{\omega}_{h}^{n}, \theta_{1 h}^{n}, \theta_{2 h}^{n}\right\}$ is zero. Then, (4.2) admits a unique solution. 


\section{Error estimate}

Now, we will obtain some a priori estimates for the difference between the exact solution and the numerical solution.

Theorem 5.1. There exists a positive constant $C$, independent of the discretization parameters $h$ and $\Delta t$, such that for all $\left\{\zeta_{h}^{i}, \chi_{h}^{i}, \xi_{h}^{i}, \eta_{1 h}^{i}, \eta_{2 h}^{i}\right\}_{i=0}^{n} \subset S_{h}$,

$$
\begin{aligned}
\| \tilde{\varphi}^{n}- & \tilde{\varphi}_{h}^{n}\left\|^{2}+\right\| \tilde{\psi}^{n}-\tilde{\psi}_{h}^{n}\left\|^{2}+\right\| \tilde{\omega}^{n}-\tilde{\omega}_{h}^{n}\left\|^{2}+\right\| \psi_{x}^{n}-\psi_{h x}^{n} \|^{2} \\
& +\left\|\varphi_{x}^{n}+\psi^{n}+l \omega^{n}-\left(\varphi_{h x}^{n}+\psi_{h}^{n}+l \omega_{h}^{n}\right)\right\|^{2} \\
& +\left\|\omega_{x}^{n}-l \varphi^{n}-\left(\omega_{h x}^{n}-l \varphi_{h}^{n}\right)\right\|^{2}+\left\|\theta_{1}^{n}-\theta_{1 h}^{n}\right\|^{2}+\left\|\theta_{2}^{n}-\theta_{2 h}^{n}\right\|^{2} \\
\leq C & \Delta t \sum_{i=1}^{n}\left(\left\|\tilde{\varphi}_{t}^{i}-\frac{1}{\Delta t}\left(\tilde{\varphi}^{i}-\tilde{\varphi}^{i-1}\right)\right\|^{2}+\left\|\tilde{\psi}_{t}^{i}-\frac{1}{\Delta t}\left(\tilde{\psi}^{i}-\tilde{\psi}^{i-1}\right)\right\|^{2}\right. \\
& +\left\|\tilde{\omega}_{t}^{i}-\frac{1}{\Delta t}\left(\tilde{\omega}^{i}-\tilde{\omega}^{i-1}\right)\right\|^{2}+\left\|\theta_{1 t}^{i}-\frac{1}{\Delta t}\left(\theta_{1}^{i}-\theta_{1}^{i-1}\right)\right\|^{2} \\
& +\left\|\theta_{2 t}^{i}-\frac{1}{\Delta t}\left(\theta_{2}^{i}-\theta_{2}^{i-1}\right)\right\|^{2}+\left\|\tilde{\varphi}^{i}-\zeta_{h}^{i}\right\|^{2}+\left\|\tilde{\varphi}_{x}^{i}-\zeta_{h x}^{i}\right\|^{2} \\
& +\left\|\tilde{\psi}^{i}-\chi_{h}^{i}\right\|^{2}+\left\|\tilde{\psi}_{x}^{i}-\chi_{h x}^{i}\right\|^{2}+\left\|\tilde{\omega}^{i}-\xi_{h}^{i}\right\|^{2}+\left\|\tilde{\omega}_{h x}^{i}-\xi_{h x}^{i}\right\|^{2} \\
& \left.+\left\|\theta_{1}^{i}-\eta_{1 h}^{i}\right\|^{2}+\left\|\theta_{1 x}^{i}-\eta_{1 h x}^{i}\right\|^{2}+\left\|\theta_{2}^{i}-\eta_{2 h}^{i}\right\|^{2}+\left\|\theta_{2 x}^{i}-\eta_{2 h x}^{i}\right\|^{2}\right) \\
& C \sum^{n-1}\left(\left\|\tilde{\varphi}^{i}-\zeta_{h}^{i}-\left(\tilde{\varphi}^{i+1}-\zeta_{h}^{i+1}\right)\right\|^{2}+\left\|\tilde{\psi}^{i}-\chi_{h}^{i}-\left(\tilde{\psi}^{i+1}-\chi_{h}^{i+1}\right)\right\|^{2}\right. \\
+ & \frac{t}{i=1} \sum^{i}\left(\left\|\tilde{\omega}^{i}-\xi_{h}^{i}-\left(\tilde{\omega}^{i+1}-\xi_{h}^{i+1}\right)\right\|^{2}+\left\|\theta_{1}^{i}-\theta_{1 h}^{i}-\left(\theta_{1}^{i+1}-\eta_{1 h}^{i+1}\right)\right\|^{2}\right. \\
& \left.+\left\|\theta_{2}^{i}-\theta_{2 h}^{i}-\left(\theta_{2}^{i+1}-\eta_{2 h}^{i+1}\right)\right\|^{2}\right) \\
+ & C\left(\left\|\varphi^{1}-\tilde{\varphi}_{h}^{0}\right\|^{2}+\left\|\psi^{1}-\tilde{\psi}_{h}^{0}\right\|^{2}+\left\|\omega^{1}-\tilde{\omega}_{h}^{0}\right\|^{2}+\left\|\psi^{0}-\psi_{h}^{0}\right\|^{2}\right. \\
& +\left\|\varphi_{x}^{0}+\psi^{0}+l \omega^{0}-\left(\varphi_{h x}^{0}+\psi_{h}^{0}+l \omega_{h}^{0}\right)\right\|^{2} \\
& \left.+\left\|\omega_{x}^{0}-l \varphi^{0}-\left(\omega_{h x}^{0}-l \varphi_{h}^{0}\right)\right\|^{2}+\left\|\theta_{1}^{0}-\theta_{1 h}^{0}\right\|^{2}+\left\|\theta_{2}^{0}-\theta_{2 h}^{0}\right\|^{2}\right) . \\
& (\|)
\end{aligned}
$$

Proof. The proof is divided into three steps to help the reader follow the arguments and the calculations.

Step 1. For a continuous function $v(t)$, let $v^{n}=v\left(t_{n}\right)$. Subtracting variational equation (4.1) $)_{1}$, at time $t_{n}$ for $\zeta=\zeta_{h} \in S_{h}$, and the discrete variational equation $(4.2)_{1}$ results in

$$
\begin{aligned}
& \rho_{1}\left(\tilde{\varphi}_{t}^{n}-\frac{1}{\Delta t}\left(\tilde{\varphi}_{h}^{n}-\tilde{\varphi}_{h}^{n-1}\right), \zeta_{h}\right)+k\left(\varphi_{x}^{n}+\psi^{n}+l \omega^{n}-\left(\varphi_{h x}^{n}+\psi_{h}^{n}+l \omega_{h}^{n}\right), \zeta_{h x}\right) \\
& \quad+\gamma_{1}\left(\tilde{\varphi}_{x}^{n}+\tilde{\psi}^{n}+l \tilde{\omega}^{n}-\left(\tilde{\varphi}_{h x}^{n}+\tilde{\psi}_{h}^{n}+l \tilde{\omega}_{h}^{n}\right), \zeta_{h x}\right) \\
& \quad-k_{0} l\left(\omega_{x}^{n}-l \varphi^{n}-\left(\omega_{h x}^{n}-l \varphi_{h}^{n}\right), \zeta_{h}\right)-\gamma_{0} l\left(\tilde{\omega}_{x}^{n}-l \tilde{\varphi}^{n}-\left(\tilde{\omega}_{h x}^{n}-l \tilde{\varphi}_{h}^{n}\right), \zeta_{h}\right) \\
& \quad+l \alpha\left(\theta_{1}^{n}-\theta_{1 h}^{n}, \zeta_{h}\right)=0 .
\end{aligned}
$$


Thus,

$$
\left\{\begin{array}{c}
\rho_{1}\left(\tilde{\varphi}_{t}^{n}-\frac{1}{\Delta t}\left(\tilde{\varphi}_{h}^{n}-\tilde{\varphi}_{h}^{n-1}\right), \tilde{\varphi}^{n}-\tilde{\varphi}_{h}^{n}\right) \\
\quad+k\left(\varphi_{x}^{n}+\psi^{n}+l \omega^{n}-\left(\varphi_{h x}^{n}+\psi_{h}^{n}+l \omega_{h}^{n}\right), \tilde{\varphi}_{x}^{n}-\tilde{\varphi}_{h x}^{n}\right) \\
\quad+\gamma_{1}\left(\tilde{\varphi}_{x}^{n}+\tilde{\psi}^{n}+l \tilde{\omega}^{n}-\left(\tilde{\varphi}_{h x}^{n}+\tilde{\varphi}_{h}^{n}+l \tilde{\omega}_{h}^{n}\right), \tilde{\varphi}_{x}^{n}-\tilde{\varphi}_{h x}^{n}\right) \\
\quad-k_{0} l\left(\omega_{x}^{n}-l \varphi^{n}-\left(\omega_{h x}^{n}-l \varphi_{h}^{n}\right), \tilde{\varphi}^{n}-\tilde{\varphi}_{h}^{n}\right) \\
\quad-\gamma_{0} l\left(\tilde{\omega}_{x}^{n}-l \tilde{\varphi}^{n}-\left(\tilde{\omega}_{h x}^{n}-l \tilde{\varphi}_{h}^{n}\right), \tilde{\varphi}^{n}-\tilde{\varphi}_{h}^{n}\right)+l \alpha\left(\theta_{1}^{n}-\theta_{1 h}^{n}, \tilde{\varphi}^{n}-\tilde{\varphi}_{h}^{n}\right) \\
=\rho_{1}\left(\tilde{\varphi}_{t}^{n}-\frac{1}{\Delta t}\left(\tilde{\varphi}_{h}^{n}-\tilde{\varphi}_{h}^{n-1}\right), \tilde{\varphi}^{n}-\zeta_{h}\right) \\
\quad+k\left(\varphi_{x}^{n}+\psi^{n}+l \omega^{n}-\left(\varphi_{h x}^{n}+\psi_{h}^{n}+l \omega_{h}^{n}\right), \tilde{\varphi}_{x}^{n}-\zeta_{h x}\right) \\
\quad+\gamma_{1}\left(\tilde{\varphi}_{x}^{n}+\tilde{\psi}^{n}+l \tilde{\omega}^{n}-\left(\tilde{\varphi}_{h x}^{n}+\tilde{\varphi}_{h}^{n}+l \tilde{\omega}_{h}^{n}\right), \tilde{\varphi}_{x}^{n}-\zeta_{h x}\right) \\
\quad-k_{0} l\left(\omega_{x}^{n}-l \varphi^{n}-\left(\omega_{h x}^{n}-l \varphi_{h}^{n}\right), \tilde{\varphi}^{n}-\zeta_{h}\right) \\
\quad-\gamma_{0} l\left(\tilde{\omega}_{x}^{n}-l \tilde{\varphi}^{n}-\left(\tilde{\omega}_{h x}^{n}-l \tilde{\varphi}_{h}^{n}\right), \tilde{\varphi}^{n}-\zeta_{h}\right)+l \alpha\left(\theta_{1}^{n}-\theta_{1 h}^{n}, \tilde{\varphi}^{n}-\zeta_{h}\right) .
\end{array}\right.
$$

Now, we subtract variational equation $(4.1)_{2}$, at time $t_{n}$ for $\chi=\chi_{h} \in S_{h}$, and the discrete variational equation $(4.2)_{2}$ to find

$$
\begin{aligned}
& \rho_{2}\left(\tilde{\psi}_{t}^{n}-\frac{1}{\Delta t}\left(\tilde{\psi}_{h}^{n}-\tilde{\psi}_{h}^{n-1}\right), \chi_{h}\right)+b\left(\psi_{x}^{n}-\psi_{h x}^{n}, \chi_{h x}\right)+\gamma_{2}\left(\tilde{\psi}_{x}^{n}-\tilde{\psi}_{h x}^{n}, \chi_{h x}\right) \\
& \quad+k\left(\varphi_{x}^{n}+\psi^{n}+l \omega^{n}-\left(\varphi_{h x}^{n}+\psi_{h}^{n}+l \omega_{h}^{n}\right), \chi_{h}\right) \\
& \quad+\gamma_{1}\left(\tilde{\varphi}_{x}^{n}+\tilde{\psi}^{n}+l \tilde{\omega}^{n}-\left(\tilde{\varphi}_{h x}^{n}+\tilde{\psi}_{h}^{n}+l \tilde{\omega}_{h}^{n}\right), \chi_{h}\right)-\alpha\left(\theta_{2}^{n}-\theta_{2 h}^{n}, \chi_{h x}\right)=0 .
\end{aligned}
$$

Therefore,

$$
\left\{\begin{aligned}
\rho_{2}( & \left.\tilde{\psi}_{t}^{n}-\frac{1}{\Delta t}\left(\tilde{\psi}_{h}^{n}-\tilde{\psi}_{h}^{n-1}\right), \tilde{\psi}^{n}-\tilde{\psi}_{h}^{n}\right)+b\left(\psi_{x}^{n}-\psi_{h x}^{n}, \tilde{\psi}_{x}^{n}-\tilde{\psi}_{h x}^{n}\right) \\
& +\gamma_{2}\left(\tilde{\psi}_{x}^{n}-\tilde{\psi}_{h x}^{n}, \tilde{\psi}_{x}^{n}-\tilde{\psi}_{h x}^{n}\right) \\
& +k\left(\varphi_{x}^{n}+\psi^{n}+l \omega^{n}-\left(\varphi_{h x}^{n}+\psi_{h}^{n}+l \omega_{h}^{n}\right), \tilde{\psi}^{n}-\tilde{\psi}_{h}^{n}\right) \\
& +\gamma_{1}\left(\tilde{\varphi}_{x}^{n}+\tilde{\psi}^{n}+l \tilde{\omega}^{n}-\left(\tilde{\varphi}_{h x}^{n}+\tilde{\psi}_{h}^{n}+l \tilde{\omega}_{h}^{n}\right), \tilde{\psi}^{n}-\tilde{\psi}_{h}^{n}\right) \\
& -\alpha\left(\theta_{2}^{n}-\theta_{2 h}^{n}, \tilde{\psi}_{x}^{n}-\tilde{\psi}_{h x}^{n}\right) \\
= & \rho_{2}\left(\tilde{\psi}_{t}^{n}-\frac{1}{\Delta t}\left(\tilde{\psi}_{h}^{n}-\tilde{\psi}_{h}^{n-1}\right), \tilde{\psi}^{n}-\chi_{h}\right)+b\left(\psi_{x}^{n}-\psi_{h x}^{n}, \tilde{\psi}_{x}^{n}-\chi_{h x}\right) \\
& +\gamma_{2}\left(\tilde{\psi}_{x}^{n}-\tilde{\psi}_{h x}^{n}, \tilde{\psi}_{x}^{n}-\chi_{h x}\right) \\
& +k\left(\varphi_{x}^{n}+\psi^{n}+l \omega^{n}-\left(\varphi_{h x}^{n}+\psi_{h}^{n}+l \omega_{h}^{n}\right), \tilde{\psi}^{n}-\chi_{h}\right) \\
& +\gamma_{1}\left(\tilde{\varphi}_{x}^{n}+\tilde{\psi}^{n}+l \tilde{\omega}^{n}-\left(\tilde{\varphi}_{h x}^{n}+\tilde{\psi}_{h}^{n}+l \tilde{\omega}_{h}^{n}\right), \tilde{\psi}^{n}-\chi_{h}\right) \\
& -\alpha\left(\theta_{2}^{n}-\theta_{2 h}^{n}, \tilde{\psi}_{x}^{n}-\chi_{h x}\right) .
\end{aligned}\right.
$$

Next, we turn to the longitudinal displacement. We subtract $(4.1)_{3}$, at time $t_{n}$ for $\xi=\xi_{h} \in S_{h}$, and the discrete variational equation (4.2) 3 to get

$$
\begin{aligned}
& \rho_{1}\left(\tilde{\omega}_{t}^{n}-\frac{1}{\Delta t}\left(\tilde{\omega}_{h}^{n}-\tilde{\omega}_{h}^{n-1}\right), \xi_{h}\right)+\gamma_{0}\left(\tilde{\omega}_{x}^{n}-l \tilde{\varphi}^{n}-\left(\tilde{\omega}_{h x}^{n}-l \tilde{\varphi}_{h}^{n}\right), \xi_{h x}\right) \\
& \quad+k l\left(\varphi_{x}^{n}+\psi^{n}+l \omega^{n}-\left(\varphi_{h x}^{n}+\psi_{h}^{n}+l \omega_{h}^{n}\right), \xi_{h}\right) \\
& \quad+\gamma_{1} l\left(\tilde{\varphi}_{x}^{n}+\tilde{\psi}^{n}+l \tilde{\omega}^{n}-\left(\tilde{\varphi}_{h x}^{n}+\tilde{\psi}_{h}^{n}+l \tilde{\omega}_{h}^{n}\right), \xi_{h}\right) \\
& \quad+k_{0}\left(\omega_{x}^{n}-l \varphi^{n}-\left(\omega_{h x}^{n}-l \varphi_{h}^{n}\right), \xi_{h x}\right)-\alpha\left(\theta_{1}^{n}-\theta_{1 h}^{n}, \xi_{h x}\right)=0 .
\end{aligned}
$$


As a consequence, we have

$$
\left\{\begin{array}{c}
\rho_{1}\left(\tilde{\omega}_{t}^{n}-\frac{1}{\Delta t}\left(\tilde{\omega}_{h}^{n}-\tilde{\omega}_{h}^{n-1}\right), \tilde{\omega}^{n}-\tilde{\omega}_{h}^{n}\right) \\
\quad+\gamma_{0}\left(\tilde{\omega}_{x}^{n}-l \tilde{\varphi}^{n}-\left(\tilde{\omega}_{h x}^{n}-l \tilde{\varphi}_{h}^{n}\right), \tilde{\omega}_{x}^{n}-\tilde{\omega}_{h x}^{n}\right) \\
\quad+k l\left(\varphi_{x}^{n}+\psi^{n}+l \omega^{n}-\left(\varphi_{h x}^{n}+\psi_{h}^{n}+l \tilde{\omega}_{h}^{n}\right), \tilde{\omega}^{n}-\tilde{\omega}_{h}^{n}\right) \\
\quad+\gamma_{1} l\left(\tilde{\varphi}_{x}^{n}+\tilde{\psi}^{n}+l \tilde{\omega}^{n}-\left(\tilde{\varphi}_{h x}^{n}+\tilde{\psi}_{h}^{n}+l \tilde{\omega}_{h}^{n}\right), \tilde{\omega}^{n}-\tilde{\omega}_{h}^{n}\right) \\
\quad+k_{0}\left(\omega_{x}^{n}-l \varphi^{n}-\left(\omega_{h x}^{n}-l \varphi_{h}^{n}\right), \tilde{\omega}_{x}^{n}-\tilde{\omega}_{h x}^{n}\right)-\alpha\left(\theta_{1}^{n}-\theta_{1 h}^{n}, \tilde{\omega}_{x}^{n}-\tilde{\omega}_{h x}^{n}\right) \\
=\rho_{1}\left(\tilde{\omega}_{t}^{n}-\frac{1}{\Delta t}\left(\tilde{\omega}_{h}^{n}-\tilde{\omega}_{h}^{n-1}\right), \tilde{\omega}^{n}-\xi_{h}\right) \\
\quad+\gamma_{0}\left(\tilde{\omega}_{x}^{n}-l \tilde{\varphi}^{n}-\left(\tilde{\omega}_{h x}^{n}-l \tilde{\varphi}_{h}^{n}\right), \tilde{\omega}_{x}^{n}-\xi_{h x}\right) \\
\quad+k l\left(\varphi_{x}^{n}+\psi^{n}+l \omega^{n}-\left(\varphi_{h x}^{n}+\psi_{h}^{n}+l \omega_{h}^{n}\right), \tilde{\omega}^{n}-\xi_{h}\right) \\
\quad+\gamma_{1} l\left(\tilde{\varphi}_{x}^{n}+\tilde{\psi}^{n}+l \tilde{\omega}^{n}-\left(\tilde{\varphi}_{h x}^{n}+\tilde{\psi}_{h}^{n}+l \tilde{\omega}_{h}^{n}\right), \tilde{\omega}^{n}-\xi_{h}\right) \\
\quad+k_{0}\left(\omega_{x}^{n}-l \varphi^{n}-\left(\omega_{h x}^{n}-l \varphi_{h}^{n}\right), \tilde{\omega}_{x}^{n}-\xi_{h x}\right)-\alpha\left(\theta_{1}^{n}-\theta_{1 h}^{n}, \tilde{\omega}_{x}^{n}-\xi_{h x}\right) .
\end{array}\right.
$$

Similar ideas applied to the variational equations to the temperatures yield

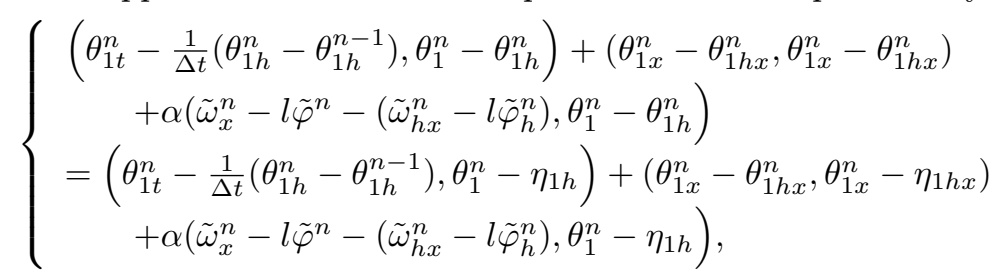

and

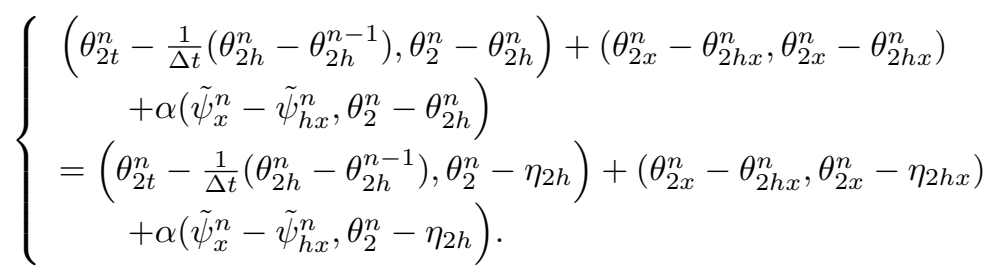

Step 2. To handle the first term in equation (5.1) we observe that

$$
\begin{aligned}
\left(\tilde{\varphi}_{t}^{n}-\right. & \left.\frac{1}{\Delta t}\left(\tilde{\varphi}_{h}^{n}-\tilde{\varphi}_{h}^{n-1}\right), \tilde{\varphi}^{n}-\tilde{\varphi}_{h}^{n}\right) \\
= & \left(\tilde{\varphi}_{t}^{n}-\frac{1}{\Delta t}\left(\tilde{\varphi}^{n}-\tilde{\varphi}^{n-1}\right), \tilde{\varphi}^{n}-\tilde{\varphi}_{h}^{n}\right) \\
& \quad+\frac{1}{\Delta t}\left(\tilde{\varphi}^{n}-\tilde{\varphi}^{n-1}-\left(\tilde{\varphi}_{h}^{n}-\tilde{\varphi}_{h}^{n-1}\right), \tilde{\varphi}^{n}-\tilde{\varphi}_{h}^{n}\right) \\
= & \left(\tilde{\varphi}_{t}^{n}-\frac{1}{\Delta t}\left(\tilde{\varphi}^{n}-\tilde{\varphi}^{n-1}\right), \tilde{\varphi}^{n}-\tilde{\varphi}_{h}^{n}\right) \\
& \quad+\frac{1}{2 \Delta t}\left\|\tilde{\varphi}^{n}-\tilde{\varphi}_{h}^{n}-\left(\tilde{\varphi}^{n-1}-\tilde{\varphi}_{h}^{n-1}\right)\right\|^{2} \\
& \left.\quad+\frac{1}{2 \Delta t}\left(\left\|\tilde{\varphi}^{n}-\tilde{\varphi}_{h}^{n}\right\|^{2}-\| \tilde{\varphi}^{n-1}-\tilde{\varphi}_{h}^{n-1}\right) \|^{2}\right) .
\end{aligned}
$$

where (4.4) was used. We deal with similar terms in (5.2)-(5.5) in the same way. 
Then, using again (4.3) and (4.4) and adding (5.1)-(5.5) we end up with

$$
\begin{aligned}
& \frac{\rho_{1}}{2 \Delta t}\left(\left\|\tilde{\varphi}^{n}-\tilde{\varphi}_{h}^{n}\right\|^{2}-\left\|\tilde{\varphi}^{n-1}-\tilde{\varphi}_{h}^{n-1}\right\|^{2}+\left\|\tilde{\omega}^{n}-\tilde{\omega}_{h}^{n}\right\|^{2}-\left\|\tilde{\omega}^{n-1}-\tilde{\omega}_{h}^{n-1}\right\|^{2}\right) \\
& +\frac{\rho_{2}}{2 \Delta t}\left(\left\|\tilde{\psi}^{n}-\tilde{\psi}_{h}^{n}\right\|^{2}-\left\|\tilde{\psi}^{n-1}-\tilde{\psi}_{h}^{n-1}\right\|^{2}\right) \\
& +\frac{k}{2 \Delta t}\left(\left\|\varphi_{x}^{n}+\psi^{n}+l \omega^{n}-\left(\varphi_{h x}^{n}+\psi_{h}^{n}+l \omega_{h}^{n}\right)\right\|^{2}\right. \\
& \left.-\left\|\varphi_{x}^{n-1}+\psi^{n-1}+l \omega^{n-1}-\left(\varphi_{h x}^{n-1}+\psi_{h}^{n-1}+l \omega_{h}^{n-1}\right)\right\|^{2}\right) \\
& +\frac{k_{0}}{\Delta t}\left(\left\|\omega_{x}^{n}-l \varphi^{n}-\left(\omega_{h x}^{n}-l \varphi_{h}^{n}\right)\right\|^{2}-\left\|\omega_{x}^{n-1}-l \varphi^{n-1}-\left(\omega_{h x}^{n-1}-l \varphi_{h}^{n-1}\right)\right\|^{2}\right) \\
& +\frac{b}{2 \Delta t}\left(\left\|\psi_{x}^{n}-\psi_{h x}^{n}\right\|^{2}-\left\|\psi_{x}^{n-1}-\psi_{h x}^{n-1}\right\|^{2}\right) \\
& +\frac{1}{2 \Delta t}\left(\left\|\theta_{1}^{n}-\theta_{1 h}^{n}\right\|^{2}-\left\|\theta_{1}^{n-1}-\theta_{1 h}^{n-1}\right\|^{2}+\left\|\theta_{2}^{n}-\theta_{2 h}^{n}\right\|^{2}-\left\|\theta_{2}^{n-1}-\theta_{2 h}^{n-1}\right\|^{2}\right) \\
& +C\left(\left\|\tilde{\psi}_{x}^{n}-\tilde{\psi}_{h x}^{n}\right\|^{2}+\left\|\tilde{\varphi}_{x}^{n}+\tilde{\psi}^{n}+l \tilde{\omega}^{n}-\left(\tilde{\varphi}_{h x}^{n}+\tilde{\psi}_{h}^{n}+l \tilde{\omega}_{h}^{n}\right)\right\|^{2}\right) \\
& +C\left(\left\|\tilde{\omega}_{x}^{n}-l \tilde{\varphi}^{n}-\left(\tilde{\omega}_{h x}^{n}-l \tilde{\varphi}_{h}^{n}\right)\right\|^{2}+\left\|\theta_{1 x}^{n}-\theta_{1 h x}^{n}\right\|^{2}+\left\|\theta_{2 x}^{n}-\theta_{2 h x}^{n}\right\|^{2}\right) \\
& \leq C\left(\left\|\tilde{\varphi}_{t}^{n}-\frac{1}{\Delta t}\left(\tilde{\varphi}^{n}-\tilde{\varphi}^{n-1}\right)\right\|^{2}+\frac{1}{\Delta t}\left(\tilde{\varphi}^{n}-\tilde{\varphi}^{n-1}-\left(\tilde{\varphi}_{h}^{n}-\tilde{\varphi}_{h}^{n-1}\right), \tilde{\varphi}^{n}-\zeta_{h}\right)\right) \\
& +C\left(\left\|\tilde{\varphi}^{n}-\tilde{\varphi}_{h}^{n}\right\|^{2}+\left\|\tilde{\varphi}^{n}-\zeta_{h}\right\|^{2}+\left\|\tilde{\varphi}_{x}^{n}-\zeta_{h x}^{2}\right\|^{2}\right) \\
& +C\left(\left\|\varphi_{x}^{n}+\psi^{n}+l \omega^{n}-\left(\varphi_{h x}^{n}+\psi_{h}^{n}+l \omega_{h}^{n}\right)\right\|^{2}\right)+\left\|\omega_{x}^{n}-l \varphi^{n}-\left(\omega_{h x}^{n}-l \varphi_{h}^{n}\right)\right\|^{2} \\
& +C\left(\left\|\theta_{1}^{n}-\theta_{1 h}^{n}\right\|^{2}+\left\|\tilde{\psi}_{t}^{n}-\frac{1}{\Delta t}\left(\tilde{\psi}^{n}-\tilde{\psi}^{n-1}\right)\right\|^{2}+\left\|\tilde{\psi}^{n}-\tilde{\psi}_{h}^{n}\right\|^{2}\right) \\
& +C\left(\frac{1}{\Delta t}\left(\tilde{\psi}^{n}-\tilde{\psi}^{n-1}-\left(\tilde{\psi}_{h}^{n}-\tilde{\psi}_{h}^{n-1}\right), \tilde{\psi}^{n}-\chi_{h}\right)+\left\|\tilde{\psi}^{n}-\chi_{h}\right\|^{2}\right) \\
& +C\left(\left\|\psi_{x}^{n}-\psi_{h x}^{n}\right\|^{2}+\left\|\tilde{\psi}_{x}^{n}-\chi_{h x}\right\|^{2}+\left\|\theta_{2}^{n}-\theta_{2 h}^{n}\right\|^{2}\right) \\
& +C\left(\left\|\tilde{\omega}_{t}^{n}-\frac{1}{\Delta t}\left(\tilde{\omega}^{n}-\tilde{\omega}^{n-1}\right)\right\|^{2}+\frac{1}{\Delta t}\left(\tilde{\omega}^{n}-\tilde{\omega}^{n-1}-\left(\tilde{\omega}_{h}^{n}-\tilde{\omega}_{h}^{n-1}\right), \tilde{\omega}^{n}-\xi_{h}\right)\right) \\
& +C\left(\left\|\tilde{\omega}^{n}-\tilde{\omega}_{h}^{n}\right\|^{2}+\left\|\tilde{\omega}^{n}-\xi_{h}\right\|^{2}+\left\|\tilde{\omega}_{h x}^{n}-\xi_{h x}\right\|^{2}\right) \\
& +C\left(\left\|\theta_{1 t}^{n}-\frac{1}{\Delta t}\left(\theta_{1}^{n}-\theta_{1}^{n-1}\right)\right\|^{2}+\frac{1}{\Delta t}\left(\theta_{1}^{n}-\theta_{1}^{n-1}-\left(\theta_{1 h}^{n}-\theta_{1 h}^{n-1}\right), \theta_{1}^{n}-\eta_{1 h}\right)\right) \\
& +C\left(\left\|\theta_{2 t}^{n}-\frac{1}{\Delta t}\left(\theta_{2}^{n}-\theta_{2}^{n-1}\right)\right\|^{2}+\frac{1}{\Delta t}\left(\theta_{2}^{n}-\theta_{2}^{n-1}-\left(\theta_{2 h}^{n}-\theta_{2 h}^{n-1}\right), \theta_{1}^{n}-\eta_{2 h}\right)\right) \\
& +C\left(\left\|\theta_{1}^{n}-\eta_{1 h}\right\|^{2}+\left\|\theta_{1 x}^{n}-\eta_{1 h x}\right\|^{2}+\left\|\theta_{2}^{n}-\eta_{2 h}\right\|^{2}+\left\|\theta_{2 x}^{n}-\eta_{2 h x}\right\|^{2}\right) .
\end{aligned}
$$


Step 3. Multiplying the latter inequality by $\Delta t$ and summing over $n$ we obtain, for all $\left\{\zeta_{h}^{i}, \chi_{h}^{i}, \xi_{h}^{i}, \eta_{1 h}^{i}, \eta_{2 h}^{i}\right\}_{i=0}^{n} \subset S_{h}$,

$$
\begin{aligned}
& \left\|\tilde{\varphi}^{n}-\tilde{\varphi}_{h}^{n}\right\|^{2}+\left\|\tilde{\psi}^{n}-\tilde{\psi}_{h}^{n}\right\|^{2}+\left\|\tilde{\omega}^{n}-\tilde{\omega}_{h}^{n}\right\|^{2}+\left\|\psi_{x}^{n}-\psi_{h x}^{n}\right\|^{2} \\
& +\left\|\varphi_{x}^{n}+\psi^{n}+l \omega^{n}-\left(\varphi_{h x}^{n}+\psi_{h}^{n}+l \omega_{h}^{n}\right)\right\|^{2} \\
& +\left\|\omega_{x}^{n}-l \varphi^{n}-\left(\omega_{h x}^{n}-l \varphi_{h}^{n}\right)\right\|^{2}+\left\|\theta_{1}^{n}-\theta_{1 h}^{n}\right\|^{2}+\left\|\theta_{2}^{n}-\theta_{2 h}^{n}\right\|^{2} \\
& \leq C \Delta t \sum_{i=1}^{n}\left(\left\|\tilde{\varphi}^{i}-\tilde{\varphi}_{h}^{i}\right\|^{2}+\left\|\tilde{\psi}^{i}-\tilde{\psi}_{h}^{i}\right\|^{2}+\left\|\tilde{\omega}^{i}-\tilde{\omega}_{h}^{i}\right\|^{2}+\left\|\psi_{x}^{i}-\psi_{h x}^{i}\right\|^{2}\right. \\
& +\left\|\varphi_{x}^{i}+\psi^{i}+l \omega^{i}-\left(\varphi_{h x}^{i}+\psi_{h}^{i}+l \omega_{h}^{i}\right)\right\|^{2} \\
& +\left\|\omega_{x}^{i}-l \varphi^{i}-\left(\omega_{h x}^{i}-l \varphi_{h}^{i}\right)\right\|^{2}+\left\|\theta_{1}^{i}-\theta_{1 h}^{i}\right\|^{2}+\left\|\theta_{2}^{i}-\theta_{2 h}^{i}\right\|^{2} \\
& +\left\|\tilde{\varphi}_{t}^{i}-\frac{1}{\Delta t}\left(\tilde{\varphi}^{i}-\tilde{\varphi}^{i-1}\right)\right\|^{2}+\frac{1}{\Delta t}\left(\tilde{\varphi}^{i}-\tilde{\varphi}^{i-1}-\left(\tilde{\varphi}_{h}^{i}-\tilde{\varphi}_{h}^{i-1}\right), \tilde{\varphi}^{i}-\zeta_{h}^{i}\right) \\
& +\left\|\tilde{\psi}_{t}^{i}-\frac{1}{\Delta t}\left(\tilde{\psi}^{i}-\tilde{\psi}^{i-1}\right)\right\|^{2}+\frac{1}{\Delta t}\left(\tilde{\psi}^{i}-\tilde{\psi}^{i-1}-\left(\tilde{\psi}_{h}^{i}-\tilde{\psi}_{h}^{i-1}\right), \tilde{\psi}^{i}-\chi_{h}^{i}\right) \\
& +\left\|\tilde{\omega}_{t}^{i}-\frac{1}{\Delta t}\left(\tilde{\omega}^{i}-\tilde{\omega}^{i-1}\right)\right\|^{2}+\frac{1}{\Delta t}\left(\tilde{\omega}^{i}-\tilde{\omega}^{i-1}-\left(\tilde{\omega}_{h}^{i}-\tilde{\omega}_{h}^{i-1}\right), \tilde{\omega}^{i}-\xi_{h}^{i}\right) \\
& +\left\|\theta_{1 t}^{i}-\frac{1}{\Delta t}\left(\theta_{1}^{i}-\theta_{1}^{i-1}\right)\right\|^{2}+\frac{1}{\Delta t}\left(\theta_{1}^{i}-\theta_{1}^{i-1}-\left(\theta_{1 h}^{i}-\theta_{1 h}^{i-1}\right), \theta_{1}^{i}-\eta_{1 h}^{i}\right) \\
& +\left\|\theta_{2 t}^{i}-\frac{1}{\Delta t}\left(\theta_{2}^{i}-\theta_{2}^{i-1}\right)\right\|^{2}+\frac{1}{\Delta t}\left(\theta_{2}^{i}-\theta_{2}^{i-1}-\left(\theta_{2 h}^{i}-\theta_{2 h}^{i-1}\right), \theta_{1}^{i}-\eta_{2 h}^{i}\right) \\
& +\left\|\tilde{\varphi}^{i}-\zeta_{h}^{i}\right\|^{2}+\left\|\tilde{\varphi}_{x}^{i}-\zeta_{h x}^{i}\right\|^{2}+\left\|\tilde{\psi}^{i}-\chi_{h}^{i}\right\|^{2}+\left\|\tilde{\psi}_{x}^{i}-\chi_{h x}^{i}\right\|^{2} \\
& +\left\|\tilde{\omega}^{i}-\xi_{h}^{i}\right\|^{2}+\left\|\tilde{\omega}_{h x}^{i}-\xi_{h x}^{i}\right\|^{2} \\
& \left.+\left\|\theta_{1}^{i}-\eta_{1 h}^{i}\right\|^{2}+\left\|\theta_{1 x}^{i}-\eta_{1 h x}^{i}\right\|^{2}+\left\|\theta_{2}^{i}-\eta_{2 h}^{i}\right\|^{2}+\left\|\theta_{2 x}^{i}-\eta_{2 h x}^{i}\right\|^{2}\right) \\
& +C\left(\left\|\varphi^{1}-\tilde{\varphi}_{h}^{0}\right\|^{2}+\left\|\psi^{1}-\tilde{\psi}_{h}^{0}\right\|^{2}+\left\|\omega^{1}-\tilde{\omega}_{h}^{0}\right\|^{2}+\left\|\psi_{x}^{0}-\psi_{h x}^{0}\right\|^{2}\right. \\
& +\left\|\varphi_{x}^{0}+\psi^{0}+l \omega^{0}-\left(\varphi_{h x}^{0}+\psi_{h}^{0}+l \omega_{h}^{0}\right)\right\|^{2} \\
& \left.+\left\|\omega_{x}^{0}-l \varphi^{0}-\left(\omega_{h x}^{0}-l \varphi_{h}^{0}\right)\right\|^{2}+\left\|\theta_{1}^{0}-\theta_{1 h}^{0}\right\|^{2}+\left\|\theta_{2}^{0}-\theta_{2 h}^{0}\right\|^{2}\right) \text {. }
\end{aligned}
$$

Taking into account that (with an equivalent result for similar terms)

$$
\begin{aligned}
& \sum_{i=1}^{n}\left(\tilde{\varphi}^{i}-\tilde{\varphi}^{i-1}-\left(\tilde{\varphi}_{h}^{i}-\tilde{\varphi}_{h}^{i-1}\right), \tilde{\varphi}^{i}-\zeta_{h}^{i}\right)=\left(\tilde{\varphi}^{n}-\tilde{\varphi}_{h}^{n}, \tilde{\varphi}^{n}-\zeta_{h}^{n}\right) \\
& \quad+\left(\tilde{\varphi}_{h}^{0}-\tilde{\varphi}^{0}, \tilde{\varphi}^{1}-\zeta_{h}^{1}\right)+\sum_{i=1}^{n-1}\left(\tilde{\varphi}^{i}-\tilde{\varphi}_{h}^{i}, \tilde{\varphi}^{i}-\zeta_{h}^{i}-\left(\tilde{\varphi}^{i+1}-\zeta_{h}^{i+1}\right)\right) \\
& \leq\left.C\left(\left\|\tilde{\varphi}^{n}-\tilde{\varphi}_{h}^{n}\right\|^{2}+\| \tilde{\varphi}^{n}-\zeta_{h}^{n}\right)\left\|^{2}+\right\| \tilde{\varphi}_{h}^{0}-\tilde{\varphi}^{0}\left\|^{2}+\right\| \tilde{\varphi}^{1}-\zeta_{h}^{1} \|^{2}\right) \\
&+C \Delta t \sum_{i=1}^{n-1}\left\|\tilde{\varphi}^{i}-\tilde{\varphi}_{h}^{i}\right\|^{2}+\frac{C}{\Delta t} \sum_{i=1}^{n-1}\left\|\tilde{\varphi}^{i}-\zeta_{h}^{i}-\left(\tilde{\varphi}^{i+1}-\zeta_{h}^{i+1}\right)\right\|^{2}
\end{aligned}
$$

and applying a discrete version of Gronwall's inequality, the result follows.

The linear convergence of the numerical method is summarized in the following corollary. 
Corollary 5.1. Suppose that the solution to the continuous problem is sufficiently regular, that is

$$
\begin{gathered}
\varphi, \psi, \omega \in H^{3}\left(0, T ; L^{2}(I)\right) \cap W^{1, \infty}\left(0, T ; H^{2}(I)\right) \cap H^{2}\left(0, T ; H^{1}(I)\right), \\
\theta_{1}, \theta_{2} \in H^{2}\left(0, T ; L^{2}(I)\right) \cap L^{\infty}\left(0, T ; H^{2}(I)\right) \cap H^{1}\left(0, T ; H^{1}(I)\right) .
\end{gathered}
$$

Then, there exists a positive constant $C$, independent of the discretization parameters $h$ and $\Delta t$, such that

$$
\begin{aligned}
\| \tilde{\varphi}\left(t_{n}\right) & -\tilde{\varphi}_{h}^{n}\left\|^{2}+\right\| \tilde{\psi}\left(t_{n}\right)-\tilde{\psi}_{h}^{n}\left\|^{2}+\right\| \tilde{\omega}\left(t_{n}\right)-\tilde{\omega}_{h}^{n}\left\|^{2}+\right\| \psi\left(t_{n}\right)-\psi_{h}^{n} \|^{2} \\
& +\left\|\varphi_{x}\left(t_{n}\right)+\psi\left(t_{n}\right)+l \omega\left(t_{n}\right)-\left(\varphi_{h x}^{n}+\psi_{h}^{n}+l \omega_{h}^{n}\right)\right\|^{2} \\
& \quad+\left\|\omega_{x}\left(t_{n}\right)-l \varphi\left(t_{n}\right)-\left(\omega_{h x}^{n}-l \varphi_{h}^{n}\right)\right\|^{2}+\left\|\theta_{1}\left(t_{n}\right)-\theta_{1 h}^{n}\right\|^{2}+\left\|\theta_{2}\left(t_{n}\right)-\theta_{2 h}^{n}\right\|^{2} \\
\leq & C\left(h^{2}+(\Delta t)^{2}\right) .
\end{aligned}
$$

Proof. The result is a consequence of estimates like

$$
\frac{1}{\Delta t} \sum_{i=1}^{n-1}\left\|\tilde{\varphi}^{i}-\zeta_{h}^{i}-\left(\tilde{\varphi}^{i+1}-\zeta_{h}^{i+1}\right)\right\|^{2} \leq C h^{2}\left\|\tilde{\varphi}_{t}\right\|_{L^{2}\left(0, T ; H^{1}(I)\right)}^{2},
$$

as proved in the work of Han, Shillor and Sofonea [5], and classical results on the approximation properties of the finite element spaces [2].

\section{Simulations}

We describe in this section the results of some numerical experiments performed to demonstrate the accuracy of the approximation and the behavior of the solution.

To find the solution of system (4.2) an iterative procedure was used. Assuming that $\tilde{\varphi}_{h}^{n-1}, \tilde{\psi}_{h}^{n-1}, \tilde{\omega}_{h}^{n-1}, \theta_{1 h}^{n-1}, \theta_{2 h}^{n-1}$ are known and setting

$$
\begin{gathered}
\varphi_{h}^{n, 0}=\varphi_{h}^{n-1}, \tilde{\varphi}_{h}^{n, 0}=\tilde{\varphi}_{h}^{n-1}, \psi_{h}^{n, 0}=\psi_{h}^{n-1}, \tilde{\psi}_{h}^{n, 0}=\tilde{\psi}_{h}^{n-1}, \\
\omega_{h}^{n, 0}=\omega_{h}^{n-1}, \tilde{\omega}_{h}^{n, 0}=\tilde{\omega}_{h}^{n-1}, \theta_{1 h}^{n, 0}=\theta_{1 h}^{n-1}, \theta_{2 h}^{n, 0}=\theta_{2 h}^{n-1}
\end{gathered}
$$

. we solved the problem

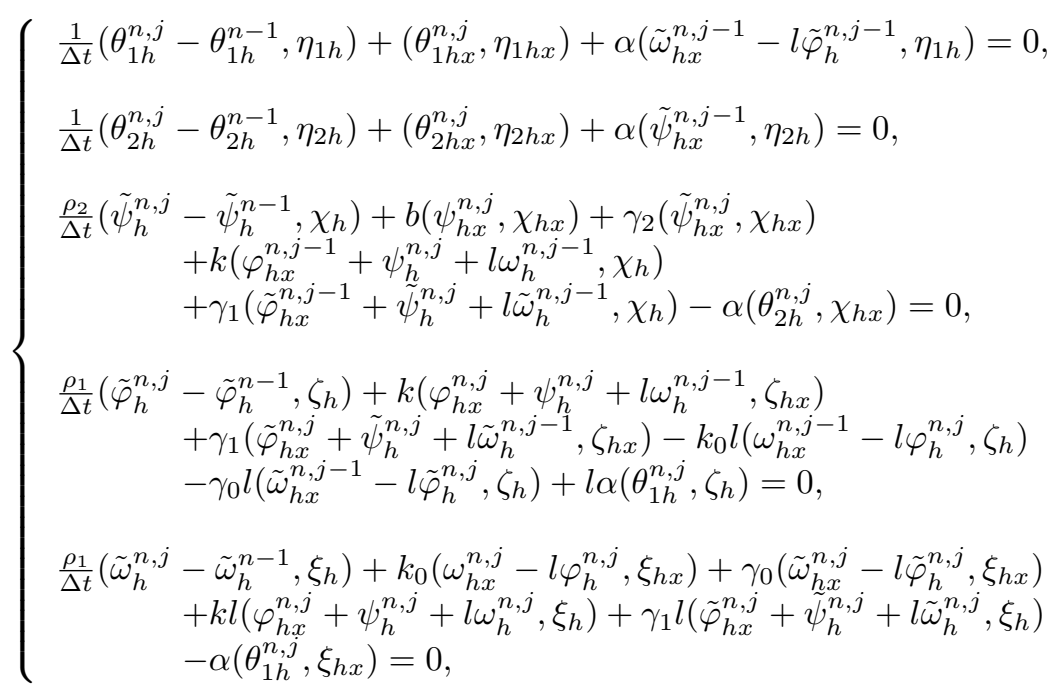

where, for $j=1,2 \cdots$,

$$
\varphi_{h}^{n, j}=\varphi_{h}^{n-1}+\Delta t \tilde{\varphi}_{h}^{n, j}, \quad \psi_{h}^{n, j}=\psi_{h}^{n-1}+\Delta t \tilde{\psi}_{h}^{n, j}, \quad \omega_{h}^{n, j}=\omega_{h}^{n-1}+\Delta t \tilde{\omega}_{h}^{n, j} .
$$


Problem (6.1) consists of five, uncoupled, linear systems of algebraic equations, with tri-diagonal matrices, that have unique solution.

A curved beam with radius of curvature $R=1$ and length $L=1$ was considered with $\rho_{1}=1, \rho_{2}=2, k=1, k_{0}=0.5$, and $b=1$. The viscosity parameters are $\gamma_{0}=\gamma_{1}=\gamma_{2}=0.1$ and the coupling constant is $\alpha=0.017$. The iterative procedure was stopped when the difference between two successive iterations became smaller than a given tolerance of $T O L=10^{-7}$.

In the first experiment, the time evolution of the system and the energy decay were investigated. The discretization parameters are $h=0.01$ and $\Delta t=10^{-4}$ and the initial data

$$
\begin{gathered}
\varphi_{0}(x)=0.01 x(x-1), \varphi_{1}(x)=20 x(x-1)^{2}, \psi_{0}(x)=\psi_{1}(x)=\omega_{0}(x)=0, \\
\omega_{1}(x)=x^{3}-x^{2}, \theta_{10}(x)=20 x \cos (0.5 \pi x), \theta_{20}(x)=10 \sin (\pi x) .
\end{gathered}
$$

As expected, the system evolved towards the zero steady-state with the energy decaying to zero very quickly. In our simulations, the temperatures $\theta_{1}$ and $\theta_{2}$ vanished faster than $\varphi, \psi$ and $\omega$. See figures 1-3.

Next, we performed a simulation to test, numerically, the error estimate. We solved the modified problem

$$
\left\{\begin{array}{c}
\rho_{1} \varphi_{t t}-k\left(\varphi_{x}+\psi+l w\right)_{x}-\gamma_{1}\left(\varphi_{x}+\psi+l w\right)_{x t}-k_{0} l\left(\omega_{x}-l \varphi\right) \\
-\gamma_{0} l\left(w_{x}-l \varphi\right)_{t}+l \alpha \theta_{1}=f_{1}, \\
\rho_{2} \psi_{t t}-b \psi_{x x}-\gamma_{2} \psi_{x x t}+k\left(\varphi_{x}+\psi+l \omega\right) \\
+\gamma_{1}\left(\varphi_{x}+l \omega+\psi\right)_{t}+\alpha \theta_{2 x}=f_{2}, \\
\rho_{1} \omega_{t t}-k_{0}\left(\omega_{x}-l \varphi\right)_{x}-\gamma_{0}\left(\omega_{x}-l \varphi\right)_{x t}+k l\left(\varphi_{x}+\psi+l \omega\right) \\
+\gamma_{1} l\left(\varphi_{x}+\psi+l \omega\right)_{t}+\alpha \theta_{1 x}=f_{3}, \\
\rho_{1} \theta_{1 t}-\theta_{1 x x}+\alpha\left(w_{x}-l \varphi\right)_{t}=f_{4}, \\
\rho_{2} \theta_{2 t}-\theta_{2 x x}+\alpha \psi_{x t}=f_{5},
\end{array}\right.
$$

where $f_{1}, f_{2}, f_{3}, f_{4}, f_{5}$, and the initial data are calculated from the exact solution

$$
\begin{aligned}
& \varphi(x, t)=-0.1\left(x^{2}-x\right) t^{2} / \sqrt{2}, \\
& \psi(x, t)=0.5 t\left(x^{2}-x\right), \\
& \omega(x, t)=t\left(x^{3}-x^{2}\right), \\
& \theta_{1}(x, t)=t^{2} \sin (\pi x), \\
& \theta_{2}(x, t)=e^{t}\left(x^{2}-x\right) .
\end{aligned}
$$

The computed errors at time $t=1.2$ are shown in table 1 where Error is defined by

$$
\begin{aligned}
& \text { Error }=\left(\left\|\tilde{\varphi}\left(t_{n}\right)-\tilde{\varphi}_{h}^{n}\right\|^{2}+\left\|\tilde{\psi}\left(t_{n}\right)-\tilde{\psi}_{h}^{n}\right\|^{2}+\left\|\tilde{\omega}\left(t_{n}\right)-\tilde{\omega}_{h}^{n}\right\|^{2}+\left\|\psi\left(t_{n}\right)-\psi_{h}^{n}\right\|^{2}\right. \\
& \quad+\left\|\varphi_{x}\left(t_{n}\right)+\psi\left(t_{n}\right)+l \omega\left(t_{n}\right)-\left(\varphi_{h x}^{n}+\psi_{h}^{n}+l \omega_{h}^{n}\right)\right\|^{2} \\
& \left.\quad+\left\|\omega_{x}\left(t_{n}\right)-l \varphi\left(t_{n}\right)-\left(\omega_{h x}^{n}-l \varphi_{h}^{n}\right)\right\|^{2}+\left\|\theta_{1}\left(t_{n}\right)-\theta_{1 h}^{n}\right\|^{2}+\left\|\theta_{2}\left(t_{n}\right)-\theta_{2 h}^{n}\right\|^{2}\right)^{\frac{1}{2}} .
\end{aligned}
$$

We note that the error decreased by a factor of 2 . The linear convergence rate can also be observed in the curves in figure 4 . 

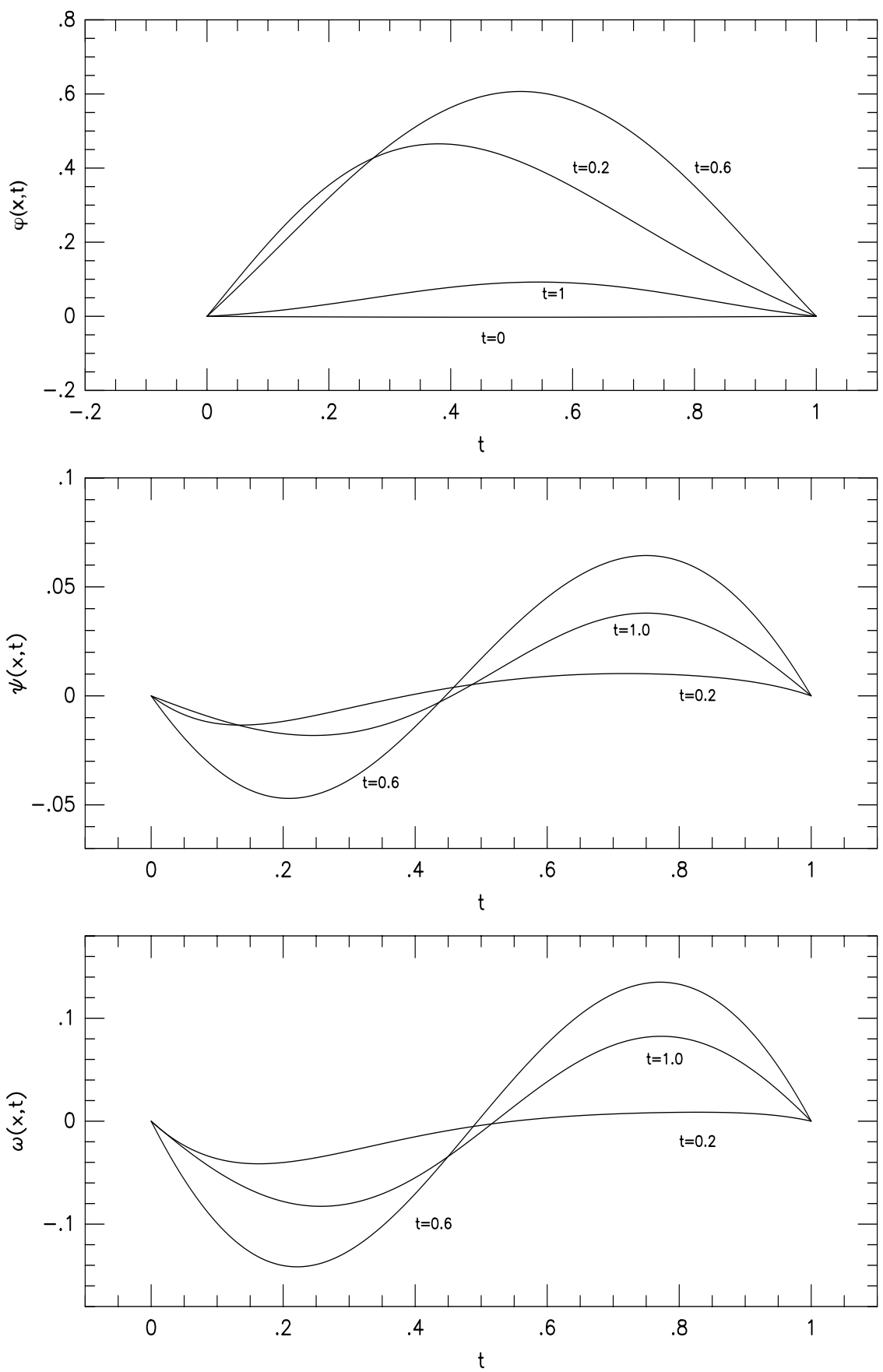

Figure 1. The evolution in time of $\varphi, \psi$, and $\omega$. 

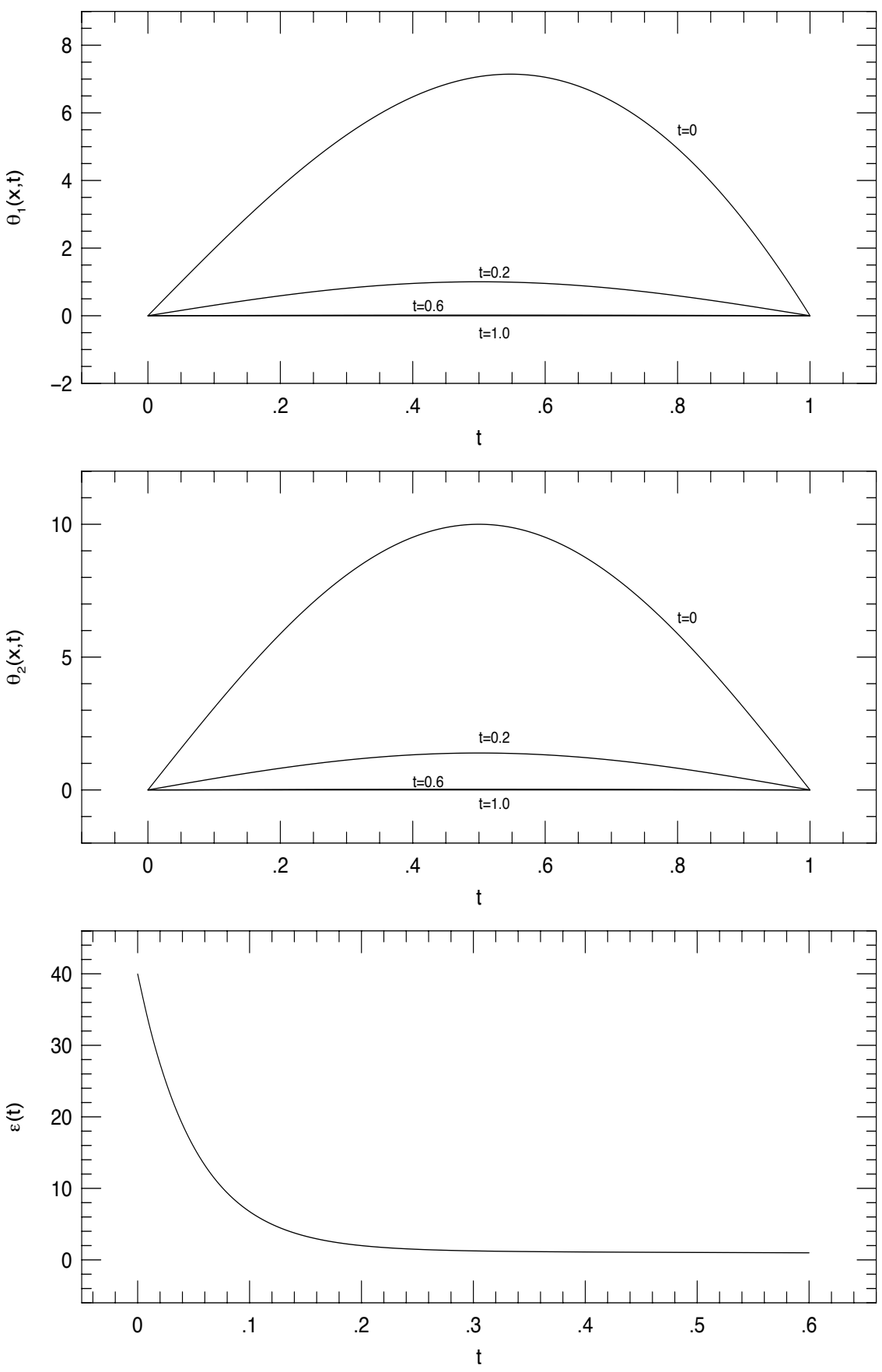

Figure 2. The evolution in time of $\theta_{1}, \theta_{2}$, and the energy. 

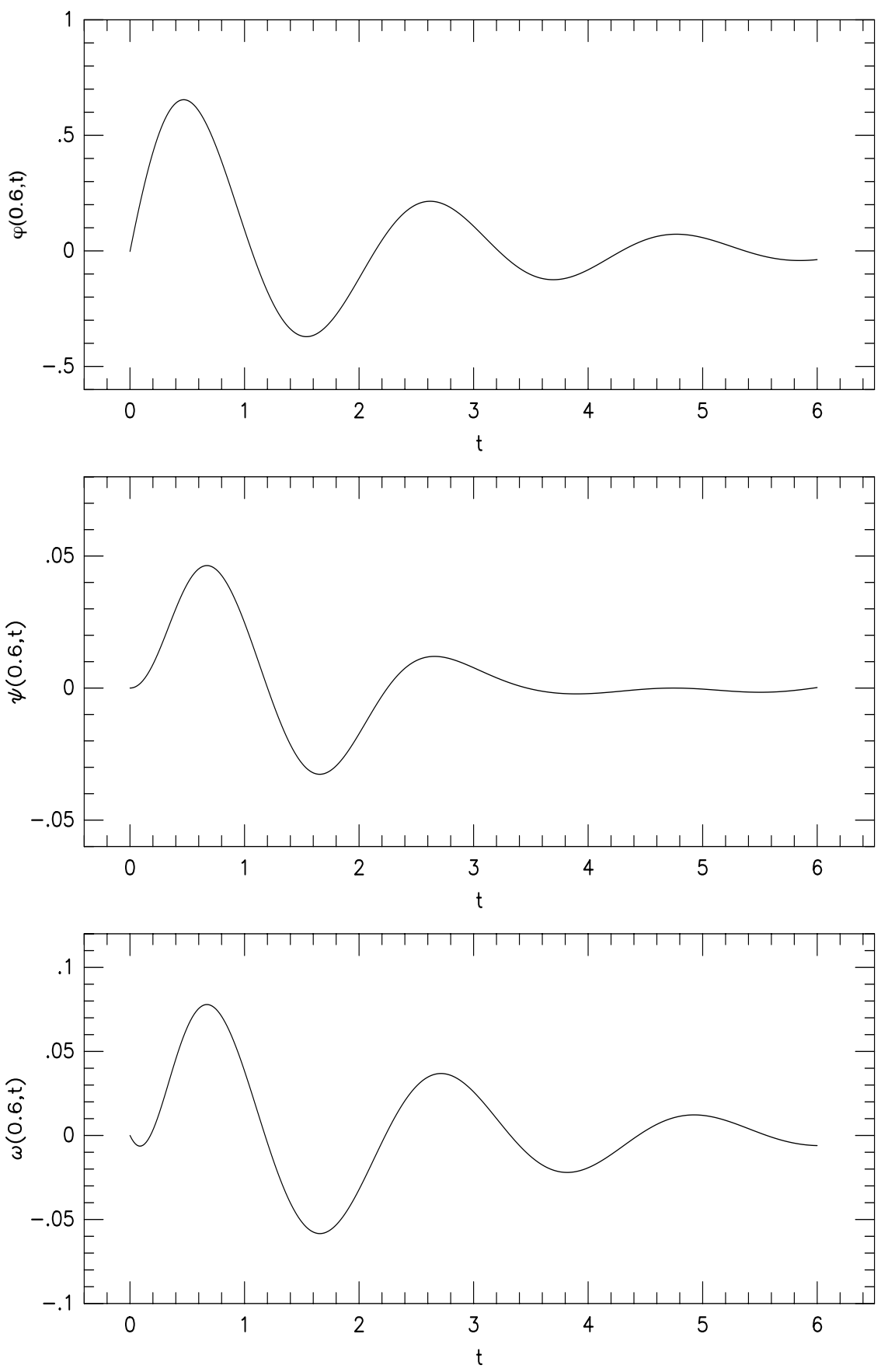

Figure 3 . The evolution in time of $\varphi, \psi$, and $\omega$ at point $x=0.6$. 

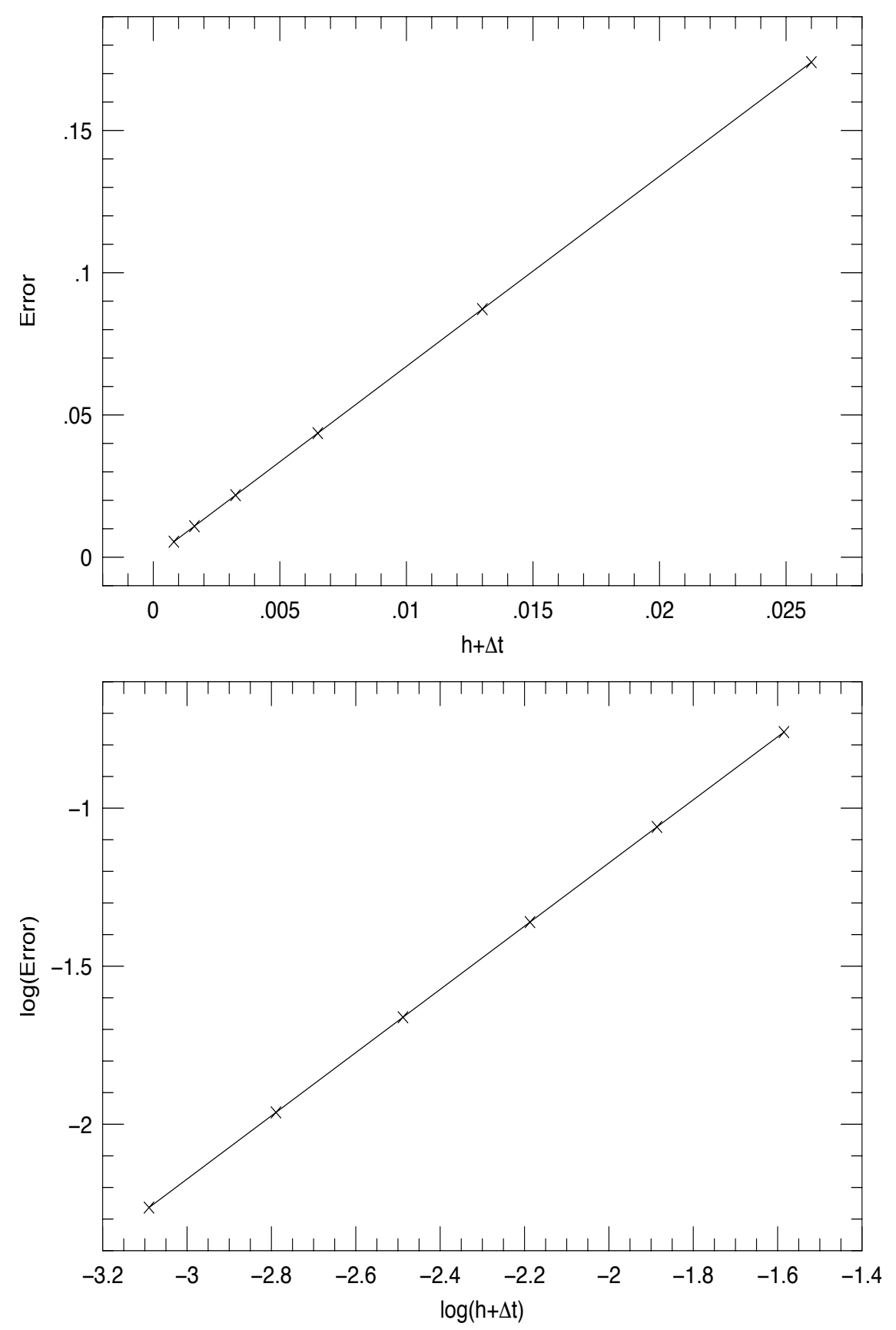

FIgURE 4. Asymptotic behavior of the numerical scheme 
TABLE 1. Computed errors when $T=1.2$.

\begin{tabular}{ccc}
\hline $\mathrm{s}$ & $\Delta t$ & Error \\
\hline 40 & $1.00 \times 10^{-3}$ & $1.74 \times 10^{-1}$ \\
80 & $5.00 \times 10^{-4}$ & $8.72 \times 10^{-2}$ \\
160 & $2.50 \times 10^{-4}$ & $4.36 \times 10^{-2}$ \\
320 & $1.25 \times 10^{-4}$ & $2.18 \times 10^{-2}$ \\
640 & $6.25 \times 10^{-5}$ & $1.09 \times 10^{-2}$ \\
1280 & $3.125 \times 10^{-5}$ & $5.45 \times 10^{-3}$ \\
\hline
\end{tabular}

\section{AcKnowledgements}

The work of M.I.M. Copetti was partially supported by the Brazilian institution CNPq (grant 304709/2017-4).

\section{REFERENCES}

[1] C. Bernardi and M. I. M. Copetti, Discretization of a nonlinear dynamic thermoviscoelastic Timoshenko beam model, Z. Angew. Math. Mech. 97, 532-549 (2017).

[2] P. G. Ciarlet, The finite element method for elliptic problems, in: P. G. Ciarlet and J. L. Lions (Eds.), Handbook of Numerical Analysis, vol. II, North Holland, 17-352, 1991.

[3] M. I. M. Copetti and J. R. Fernàndez, A dynamic contact problem involving a Timoshenko beam model, Appl. Num. Math. 63, 117-128 (2013).

[4] T. El Arwadi, A. Wehbeh and W. Youssef, Theoretical and Numerical Observability of the Bresse Beam, submitted.

[5] W. Han, M. Shillor and M. Sofonea, Variational and numerical analysis of a quasi-static viscoelastic problem with normal compliance, friction and damage, J. Comp. Appl. Math. 137, 377-398 (2001).

[6] A. Haraux, Semi-groupes lineéaires et équations d'évolution linéaires périodiques. Publication du Laboratoire d'Analyse Numérique No. 78011, Université Pierre et Marie Curie, Paris, 1978.

[7] V. Komornik, Exact controllability and stabilization. The Multiplier Method, RAM Res. Appl. Math., Masson, Paris, John Wiley, Chichester, UK, 1994.

[8] J. Lagnese, Boundary Stabilization of Thin Plates, SIAM Studies in Appl. Math., Philadelphia, 1989.

[9] F. Gallego and J. E. M. Rivera, Decay rates for solutions to thermoelastic Bresse systems of types I and III, Electron. J. Diff. Eqns. 2017, No. 73, 1-26 (2017).

[10] Z. Liu and B. Rao, Energy Decay of the thermoelastic Bresse System, Z. Angew. Math. Phys. (2009), $54-69$.

[11] Z. Liu and S. Zheng, Semigroups Associated with Dissipative Systems, 398 Research Notes in Mathematics, Chapman \& Hall/CRC, 1999.

[12] J. E. M. Rivera and R. Racke, Mildly dissipative nonlinear Timoshenko systems - global existence and exponential stability, J. Math, Anal. Appl. 276, 248-278 (2002).

[13] W. Youssef, Contrôle et stabilisation de systèmes élastiques couplés, Thesis 2009.

Maria Inês M. Copetti, LANA, Departamento de Matemática, Universidade Federal de Santa Maria, 97105-900, Santa Maria, RS, Brasil

E-mail address: mimcopetti@ufsm.br

Toufic El Arwadi, Department of mathematics and computer science, Beirut Arab University, Debbieh, Lebanon

E-mail address: t.elarwadi@bau.edu.lb

Wael Youssef, Department of mathematics, Faculty of Sciences 1, Lebanese University, Hadath, Beyrouth, Lebanon

E-mail address: Youssef.Wael@ul.edu.lb 